Authorship note: $C A B, A l$, and $J \mathrm{H}$ contributed equally to this work. RZ, TAC, and JPS contributed equally to this work.

Conflict of interest: TAC is a cofounder of Gritstone Oncology and holds equity. TAC holds equity in An2H. TAC acknowledges grant funding from Bristol Myers Squibb, AstraZeneca, Illumina, Pfizer, An2H and Eisai. TAC has served as an advisor for Bristol Myers Squibb, Illumina, Eisai, and An2H. Under a licensing agreement between NexImmune and the Johns Hopkins University, JPS is entitled to shares of royalty received by the university on sales of artificial antigen-presenting cell products described in this article. He also owns Nexlmmune stock, which is subject to certain restrictions under university policy. JPS is a member of the company's Scientific Advisory Board. The terms of this arrangement are being managed by the Johns Hopkins University in accordance with its conflict-of-interest policies. JPS acknowledges grant funding from AstraZeneca.

Copyright: (ㄷ) 2020, American Society for Clinical Investigation.

Submitted: December 30, 2019

Accepted: March 25, 2020

Published: April 23, 2020.

Reference information: /CI Insight. 2020;5(8):e135597.

https://doi.org/10.1172/jici.

insight.135597.

\section{Commensal bacteria stimulate antitumor responses via T cell cross-reactivity}

\author{
Catherine A. Bessell, ${ }^{1}$ Ariel Isser, ${ }^{2}$ Jonathan J. Havel, ${ }^{3}$ Sangyun Lee, ${ }^{4}$ David R. Bell, ${ }^{4}$ John W. Hickey, ${ }^{2}$ \\ Worarat Chaisawangwong, ${ }^{5}$ Joan Click Bieler, ${ }^{5}$ Raghvendra Srivastava, ${ }^{3}$ Fengshen Kuo, ${ }^{3}$ \\ Tanaya Purohit, ${ }^{3}$ Ruhong Zhou, ${ }^{4,6}$ Timothy A. Chan, ${ }^{3,7}$ and Jonathan P. Schneck ${ }^{1,5,9}$ \\ ${ }^{1}$ Graduate Program in Immunology and 2Department of Biomedical Engineering, Johns Hopkins University, Baltimore, \\ Maryland, USA. ${ }^{3}$ Human Oncology and Pathogenesis Program, Memorial Sloan Kettering Cancer Center, New York, New \\ York, USA. ${ }^{4}$ Computational Biology Center, IBM Thomas J. Watson Research Center, Yorktown Heights, New York, USA. \\ ${ }^{5}$ Department of Pathology, Johns Hopkins University School of Medicine, Baltimore, Maryland, USA. ${ }^{6}$ Department of \\ Chemistry, Columbia University, New York, New York, USA. ${ }^{7}$ Immunogenomics and Precision Oncology Platform, Memorial \\ Sloan Kettering Cancer Center, New York, New York, USA. ${ }^{8}$ Center for Immunotherapy and Precision Immuno-Oncology, \\ Cleveland Clinic, Cleveland, Ohio, USA. ${ }^{9}$ Institute of Cellular Engineering, Johns Hopkins University School of Medicine, \\ Baltimore, Maryland, USA.
}

\begin{abstract}
Recent studies show gut microbiota modulate antitumor immune responses; one proposed mechanism is cross-reactivity between antigens expressed in commensal bacteria and neoepitopes. We found that T cells targeting an epitope called SVYRYYGL (SVY), expressed in the commensal bacterium Bifidobacterium breve (B. breve), cross-react with a model neoantigen, SIYRYYCL (SIY). Mice lacking $B$. breve had decreased SVY-reactive T cells compared with $B$. breve-colonized mice, and the $T$ cell response was transferable by SVY immunization or by cohousing mice without Bifidobacterium with ones colonized with Bifidobacterium. Tumors expressing the model SIY neoantigen also grew faster in mice lacking $B$. breve compared with Bifidobacterium-colonized animals. B. breve colonization also shaped the SVY-reactive TCR repertoire. Finally, SVY-specific T cells recognized SIY-expressing melanomas in vivo and led to decreased tumor growth and extended survival. Our work demonstrates that commensal bacteria can stimulate antitumor immune responses via cross-reactivity and how bacterial antigens affect the $T$ cell landscape.
\end{abstract}

\section{Introduction}

The basis for differential patient responses to cancer immunotherapy involves many biological processes, including genetic variability among different patients, differences in tumor mutational load, and differential microbiome composition. Higher tumor mutational load is correlated with beneficial responses to checkpoint blockade outcomes in melanoma and non-small cell lung carcinoma (1). Mechanistically mutations in the tumor can result in novel peptides, called neoantigens, which can be targeted by $\mathrm{T}$ cells. These neoantigen-specific responses are critical for tumor clearance after checkpoint blockade immunotherapy (CPI) $(2,3)$. In addition to the quantity of potential neoantigens, several studies have also considered their quality to help identify which patients will be responsive to CPI $(4,5)$.

Another important parameter for patient outcome is the gut microbiota. Responders to checkpoint blockade have a different gut bacterial composition than nonresponders $(6,7)$; however, the potential mechanisms underlying antitumor effects are unclear. Evidence has emerged that the gut microbiota modulates the efficacy of various tumor therapies, possibly by engaging the innate and/or adaptive immune systems (8-10). Retrospective analysis of pancreatic cancer long-term survivors revealed that some high-quality neoepitope responses bear sequence homology to pathogenic or commensal bacteria (4). However, the effect of homologous epitopes between the tumor and microbiota on T cell populations is unknown. Therefore, identifying antigens expressed by microbiota and their potential cross-reactivity with $\mathrm{T}$ cells recognizing tumor-specific neoantigens will provide insight into tumor-reactive $\mathrm{T}$ cell populations that can be activated by cancer immunotherapy. In addition to identifying potential homologous antigens in microbiota, mechanistic studies must address how cross-reactive T cell populations react to tumor cells and the effect of bacterial colonization on the cross-reactive $\mathrm{T}$ cell populations. 
These studies will provide insight into how antigen mimicry between the commensal bacteria and tumor antigens can lead to beneficial antitumor responses.

Here, we identify an epitope, SVYRYYGL (SVY), found in the Bifidobacterium breve (B. breve) genome, with homology to the tumor model neoepitope antigen SIYRYYGL (SIY) expressed in B16.SIY tumors. Molecular dynamics simulations show that SVY peptide loaded on the murine $\mathrm{H} 2-\mathrm{K}^{\mathrm{b}} \mathrm{MHC}$ binds to a model TCR specific for the $\mathrm{K}^{\mathrm{b}} \mathrm{SIY}$ complex, the $2 \mathrm{C}$ transgenic TCR, but with an altered configuration. Heterogeneous $\mathrm{CD}^{+} \mathrm{T}$ cell populations, expanded from wild-type C57BL/6 animals specific for either SIY or SVY, cross-reacted with both the bacterial and tumor antigens. Indeed, Bifidobacteria colonization, as seen by differences in C57BL/ 6 mice obtained from different suppliers, The Jackson Laboratory versus Taconic Biosciences, boosted expansion of SVY-specific T cell populations in vitro, and differences in commensal bacteria influenced the SVY population in vivo. In addition, immunization of Taconic mice with the SVY antigen led to activation of SVY-specific T cells that cross-reacted and killed SIY-pulsed target cells in vivo. Furthermore, C57BL/6 mice, obtained from the different commercial sources, had differences in their antitumor response to B16.SIY tumors, which grew more slowly in Jackson than Taconic mice. C57BL/6 mice also had an altered TCR repertoire depending on their source. Finally, in an adoptive cellular therapy (ACT) treatment model, SVY-expanded T cells targeted SIY-expressing tumor cells in vivo, inhibited tumor growth, and extended survival. These studies, for the first time to our knowledge, mechanistically identify that commensal bacteria can augment antitumor immunity by amplifying T cells that cross-react with model tumor neoantigens.

\section{Results}

B. breve contains a peptide epitope (SVY) that is homologous to the SIY epitope. To identify commensal bacteria epitopes that may influence the B16.SIY antitumor response, we conducted a search of microbial genomes with the BLAST (http://www.ncbi.nlm.nih.gov/blast/), using the SIY peptide sequence as the query. Genetic alignment (Figure 1A) identified a single peptide sequence, SVYRYYGL, that varies by only 1 amino acid from the murine $\mathrm{H} 2-\mathrm{K}^{\mathrm{b}} \mathrm{MHC}$-restricted model neoepitope SIY, valine (Val) versus isoleucine (Ile) at position 2. The SVY epitope sequence, derived from the B. breve EBP, has a 126-fold stronger predicted binding constant for $\mathrm{H} 2-\mathrm{K}^{\mathrm{b}}$ compared with the next highest predicted epitope sequence from the EBP (Table 1). Processing of $B$. breve in vitro was studied by incubating heat-killed $B$. breve with splenocytes, and the resultant antigen-specific T cell expansions were analyzed (Figure 1B). By day 11, approximately $3.5 \%$ of $\mathrm{CD}^{+} \mathrm{T}$ cells were cross-reactive with the H2-K $\mathrm{K}^{\mathrm{b}}$ SIY complex (K $\mathrm{K}^{\mathrm{b}} \mathrm{SI}$ ) (Figure $1 \mathrm{~B}$ and Supplemental Figure 1; supplemental material available online with this article; https://doi.org/10.1172/jci. insight.135597DS1). In contrast, a control commensal bacterium, Lactobacillus rhamnosus, did not stimulate a $\mathrm{K}^{\mathrm{b}} \mathrm{SIY}$ cross-reactive response. Thus, $B$. breve expresses an antigen that can be processed and presented and can stimulate $\mathrm{K}^{\mathrm{b}} \mathrm{SIY}$ cross-reactive $\mathrm{CD} 8^{+} \mathrm{T}$ cells.

The biophysical interaction of the antigens was analyzed by comparing the ability of SIY and SVY to stabilize the $\mathrm{K}^{\mathrm{b}}$ MHC complex on RMA-S cells. Both SVY and SIY peptides stabilized the H2-K $\mathrm{K}^{\mathrm{b}} \mathrm{MHC}$ molecule to a similar extent, with half-maximal stabilization seen at approximately $100 \mathrm{nM}$ (Figure 1C), indicating that SIY and SVY bind $\mathrm{H} 2-\mathrm{K}^{\mathrm{b}} \mathrm{MHC}$ with equal affinity. Using $\mathrm{T}$ cells from the $2 \mathrm{C}$ transgenic mouse, which are specific for the $\mathrm{K}^{\mathrm{b}} \mathrm{SIY}$ peptide MHC (pMHC) complex, we found that this model TCR was cross-reactive with the $\mathrm{K}^{\mathrm{b} S V Y}$ complex (Figure 1D), and 2C lymphocytes proliferated equally well as $\mathrm{K}^{\mathrm{b}} \mathrm{SIY}$ and $\mathrm{K}^{\mathrm{b}} \mathrm{SVY}$ (Supplemental Figure 2). Quantitative assessment of TCR affinity, using a competitive off-rate assay, showed that $\mathrm{K}^{\mathrm{b}} \mathrm{SVY}$ has an approximately 4 -fold lower affinity, $\mathrm{k}_{\text {off }} 12.59 \mathrm{e}-4 / \mathrm{s}$, compared with 3.12e-4/s (Figure 1E), and functionally lower trends for cytokine production, TNF- $\alpha$, and IL-2, and CD107a expression were seen (Supplemental Figure 3, A-C). Thus, in a model 2C TCR system, there is cross-reactivity due to the single amino acid change, which still results in TCR binding, proliferation, and cytokine production, albeit at lower levels.

Modeling the interaction between $K^{b} S V Y$ and $K^{b} S I Y$ with the $2 C T C R$. We investigated the change in $\mathrm{K}^{\mathrm{b}}$-peptideTCR binding for the Val-to-Ile mutation at position 2 in the epitope sequence using molecular dynamics (MD) simulation. Figure 2A shows the constructed 3-way binding complex HLA-epitope-TCR, using the published individual x-ray structures of the $\mathrm{K}^{\mathrm{b}}$-epitope and TCR $(10,11)$ (see more details in Methods). Although the 2 epitope sequences differ only in the second position, the root mean square deviation (RMSD) per residue calculated between the highest populated binding pose of each epitope shows that Tyr3 (4.6 ̊), Arg4 (3.6 $\AA$ ), and Leu8 (4.3 ̊) deviate more than the mutated residue (Ile2/Val2) (2.9 A) (see Supplemental Table 1). 
A

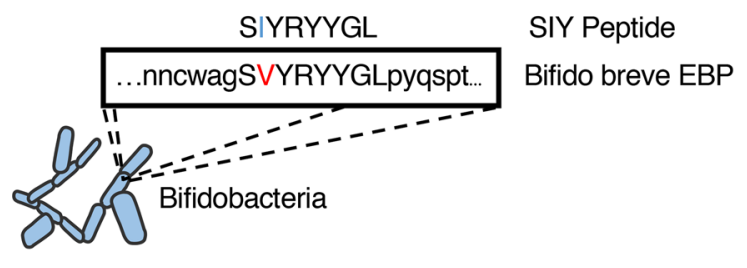

B

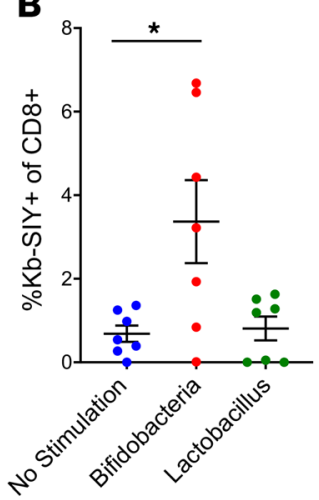

C

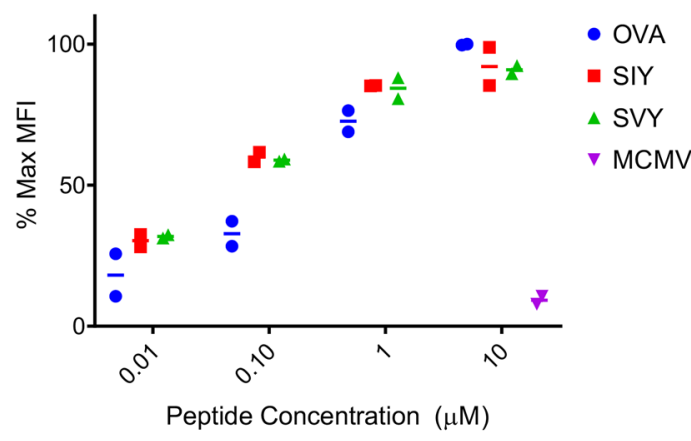

D

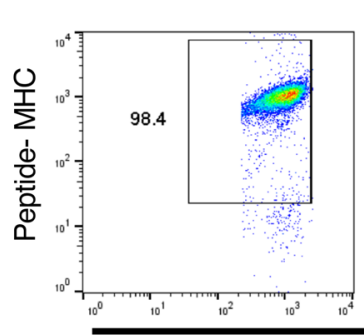

KbSVY

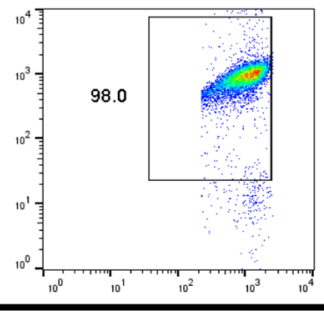

KbOVA

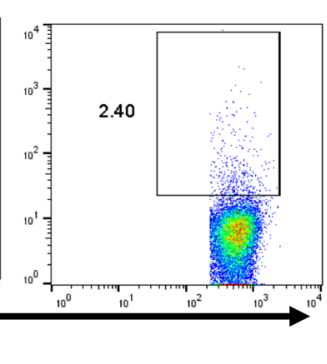

E

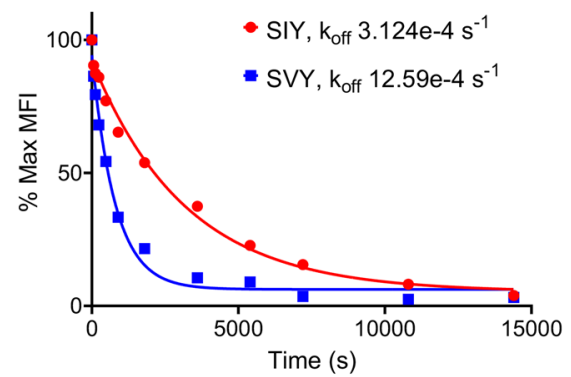

Figure 1. Commensal bacteria B. breve harbors the $\mathrm{CDB}^{+}$T cell epitope SVY. (A) Genetic map of B. breve, highlighting the homology of a peptide derived from $B$. breve exopolysaccharide biosynthesis protein (EBP) to the model epitope $K^{b} S I Y$. (B) Jackson mice splenocytes and mesenteric lymph node cells were cultured with or without heat-killed bacteria and tested for SIY-specific T cell expansion by staining with SIY peptide-loaded $\mathrm{K}^{\mathrm{b}}$-Ig dimer on day 11. Live CD8 ${ }^{+}$ lymphocytes were analyzed by flow cytometry for $\mathrm{K}^{\mathrm{b}} \mathrm{SIY}$ binding, with frequency determined by subtracting unloaded $\mathrm{K}^{\mathrm{b}}$-Ig staining frequency. $P$ value $=0.011$ by 1-way ANOVA and Dunnett's post hoc test for multiple comparisons. $N=7$. Data represent mean \pm SEM. (C) MHC stabilization assay: RMA-S cells were incubated overnight with peptide as indicated. Cell surface expression of $\mathrm{H} 2-\mathrm{K}^{\mathrm{b}}$ was determined by flow cytometry. Reported values are relative to the $\mathrm{H} 2-\mathrm{K}^{\mathrm{b}}$ mean fluorescence intensity (MFI) observed with $10 \mu \mathrm{M}$ OVA peptide. $\mathrm{mCMV}$, a non- $\mathrm{K}^{\mathrm{b}}$-restricted peptide, was used as a negative control. Data trended toward no difference between SIY and SVY groups. Data trended with no difference between SIY and SVY groups. $N=2$. Data represent mean. (D) CD8 $8^{+}$cells were isolated from the spleens of 2 C TCR (SIY-reactive) transgenic mice and stained with $1 \mu$ g of cognate $K^{\mathrm{b}} \mathrm{SIY}$-lg, cross-reactive $\mathrm{K}^{\mathrm{b}} \mathrm{SVY}$-Ig, or irrelevant $\mathrm{K}^{\mathrm{b}} \mathrm{OVA}$-Ig. Representative data shown from 1 of 3 separate experiments. (E) Competitive off-rate binding assay of $2 C$ CD $8^{+} T$ cells with $K^{b} S I Y$ or $K^{b} S V Y$ peptide MHC dimer over time by the addition of 1B2 TCR-binding antibody. Cells were gated on CD8 $8^{+}$. Cells were stained with $\mathrm{K}^{\mathrm{b}} \mathrm{OVA}$ as a negative control or experimental pMHC to gate on antigen-specific cells over time. This competitive binding assay was performed twice, with similar $\mathrm{k}_{\text {off }}$ rates determined each time.

The overlapping binding poses of $\mathrm{K}^{\mathrm{b} S I Y}$ and $\mathrm{K}^{\mathrm{b}} \mathrm{SVY}$ show that the side chains of the 3 residues with larger RMSDs deviate between the 2 epitopes (Supplemental Figure 4). Although the binding poses of the 2 epitopes differ from each other, the binding poses from the same epitope are generally consistent among the top 5 populated clusters, which account for over $98 \%$ of the MD trajectories (Supplemental Figure 5, A and B). A contact analysis found that the contacts at the interface between the peptide epitope, $\mathrm{K}^{\mathrm{b}}$, and TCR in the 3-way complex vary between epitopes because of conformational changes (Supplemental Figure 6, A and C). The frequencies of either $\mathrm{K}^{\mathrm{b}}$ or TCR atoms, making close contact (less than $6 \AA$ ) with the epitopes in the MD trajectories, are presented on the surfaces of $\mathrm{K}^{\mathrm{b}}$ and TCR (Supplemental Figure 6, A-D). The difference in SIY and SVY contact frequency (Figure $2 \mathrm{~B}$ ) shows the contact frequency on both the $\mathrm{K}^{\mathrm{b}}$ and TCR surfaces. The contact frequency difference is greater than $80 \%$ at several regions on the TCR-epitope interface, specifically where TCR atoms contact Tyr3, Arg4, and Leu8 of the epitope. These data are consistent with the RMSD analysis, shown in Supplemental Table 1, indicating that the structures of the 2 epitopes deviate the most at these residues (Figure 2C). Meanwhile, the contact profile at the $\mathrm{K}^{\mathrm{b}}$-epitope interface is more similar between epitopes, without the large differences observed at the TCR-epitope interface, indicating the altered peptide configuration is mainly on the TCR side.

We next computed, using the free energy perturbation (FEP) method, the binding affinity for each of 2 intermediate steps in the formation of the ternary complex, where each epitope is loaded onto the $\mathrm{K}^{\mathrm{b}}$ protein, and then the $\mathrm{K}^{\mathrm{b}}$-epitope binary complex binds to the TCR. We found a change in binding affinity (SVY $\rightarrow$ SIY) of $0.09 \pm 0.36$ and $-1.2 \pm 0.2 \mathrm{kcal} / \mathrm{mol}$ for the epitope loaded into $\mathrm{K}^{\mathrm{b}}$, and the subsequent binding to TCR, respectively (Figure 2D). The FEP calculation results indicate that the binding affinity of the SVY 
Table 1. IEDB predictions for Bifidobacteria EBP

\begin{tabular}{cccc}
\hline Rank & Peptide & Processing score & H2-K $^{\text {b }}$ binding $(\mathbf{n m})$ \\
1 & SVYRYYCL & 1.93 & 2.6 \\
2 & RHYIVSTL & 2.11 & 328.8 \\
3 & CGLYFPGF & 2.23 & 714.6 \\
\hline
\end{tabular}

The top 3 predicted $\mathrm{H} 2-K^{\mathrm{b}}$-restricted epitopes from the EBP using the Immune Epitope Database (IEDB) prediction algorithm.

binding to $\mathrm{H} 2-\mathrm{K}^{\mathrm{b}} \mathrm{MHC}$ is similar to that of the SIY binding; however, the binding affinity of the $\mathrm{K}^{\mathrm{b} S V Y}$ complex to TCR is somewhat weaker than $\mathrm{K}^{\mathrm{b} S I Y}$ (about $2 \mathrm{k}_{\mathrm{B}} \mathrm{T}$, the Boltzmann factor times temperature, or equivalent to $\sim 7.6$-fold difference in the binding equilibrium constant at room temperature). These results are in excellent agreement with the biophysical and functional data shown in Figure 1, C and E, indicating that epitopes indeed have similar binding affinity for $\mathrm{K}^{\mathrm{b}}$, but $\mathrm{K}^{\mathrm{b}} \mathrm{SVY}$ has somewhat weaker interactions with the $2 \mathrm{C}$ TCR than $\mathrm{K}^{\mathrm{b}}$ SIY because of the altered peptide configuration. Thus, the processing and presentation of EBP from $B$. breve leads to an SIY cross-reactive response, and in fact the SVY antigen can load nicely onto the MHC molecule. However, the amino acid substitution alters TCR recognition, creating slightly lower biophysical binding of 2C TCR to $\mathrm{pMHC}$, which may affect T cell population cross-reactivity.

$K^{b} S I Y$ - and $K^{b} S V Y$-specific $T$ cell populations are cross-reactive. To study cross-reactivity in endogenous, heterogeneous $\mathrm{T}$ cell populations, we analyzed SVY- and SIY-specific $\mathrm{T}$ cells from naive mice. $\mathrm{CD} 8^{+} \mathrm{T}$ cells from mice were enriched and stimulated with $\mathrm{K}^{\mathrm{b}} \mathrm{SIY}$ - or $\mathrm{K}^{\mathrm{b}} \mathrm{SVY}$-based artificial antigen-presenting cells (aAPCs). The resultant day 7 cultures represent a heterogeneous mix of $\mathrm{CD} 8^{+} \mathrm{T}$ cells specific for each individual antigen, as previously described (12) (see Figure 3A). Expansion with K ${ }^{\mathrm{b} S I Y}$ aAPCs led to approximately $50.4 \% \mathrm{~K}^{\mathrm{b}}$ SIY-specific $\mathrm{T}$ cells as seen by cognate $\mathrm{K}^{\mathrm{b}} \mathrm{SIY}$ dimer staining (Figure 3B, top row, left). These were cross-reactive with $\mathrm{K}^{\mathrm{b}} \mathrm{SVY}$ dimer, which had a similar 49.8\% staining and an average expansion frequency of $36.4 \%$ (Figure 3B, top row, middle; and Supplemental Figure 7A). MFIs of the antigen-reactive populations were 685 for the cognate $\mathrm{K}^{\mathrm{b}} \mathrm{SIY}$ dimer and 549 (i.e., $\sim 20 \%$ lower) for the cross-reactive response to $\mathrm{K}^{\mathrm{b}} \mathrm{SVY}$ pMHC (Figure 3B, top row). Background noncognate staining was less than 1\%. Similarly, expansion of $\mathrm{T}$ cells with $\mathrm{K}^{\mathrm{b}} \mathrm{SVY}$ aAPCs led to a cognate $\mathrm{K}^{\mathrm{b} S V Y}$-bound $\mathrm{T}$ cell population of $58.1 \%$ and a cross-reactive $\mathrm{K}^{\mathrm{b}} \mathrm{SIY}$-bound population of $54.4 \%$ of $\mathrm{CD} 8^{+} \mathrm{T}$ cells with similar MFIs and an average expansion frequency of $31.7 \%$ (Figure 3B, lower row, and Supplemental Figure 7A).

Antigen-specific $\mathrm{T}$ cells were stained with varying concentrations of $\mathrm{K}^{\mathrm{b}}$-Ig molecules to determine the $\mathrm{IC}_{50}$ of the expanded T cells for each peptide-MHC complex and overall T cell avidity (Supplemental Figure $7 \mathrm{~B}$ and Figure $3 \mathrm{C}$ ). Similar to the findings from the $2 \mathrm{C}$ transgenic $\mathrm{T}$ cells, $\mathrm{K}^{\mathrm{b}} \mathrm{SIY}$-expanded $\mathrm{T}$ cells had a higher $\mathrm{IC}_{50}(0.1 \mu \mathrm{g})$ for $\mathrm{K}^{\mathrm{b} S V Y}$ compared with $\mathrm{K}^{\mathrm{b}} \mathrm{SIY}(0.01 \mu \mathrm{g})$ (Figure $3 \mathrm{C}$, top). Differences in overall TCR affinity were also seen functionally as $\mathrm{K}^{\mathrm{b}} \mathrm{SIY}$-expanded $\mathrm{T}$ cells exhibited a lower trend of cytokine production for the cross-reacting $\mathrm{K}^{\mathrm{b}} \mathrm{SVY}$ antigen restimulation (15\% IL-2) than for the cognate SIY-pulsed RMA-S cells (30\% IL-2) (Supplemental Figure 7C), but this was not significantly different.

Interestingly, T cells expanded with $\mathrm{K}^{\mathrm{b}} \mathrm{SVY}$-based aAPCs had equal affinity $(0.1 \mu \mathrm{g})$ for both $\mathrm{K}^{\mathrm{b}} \mathrm{SVY}$ and $\mathrm{K}^{\mathrm{b}} \mathrm{SIY}$ pMHC-Ig complexes (Figure 3C, lower), and $\mathrm{K}^{\mathrm{b}} \mathrm{SVY}$-expanded $\mathrm{T}$ cells produced similar amounts of cytokines in response to both SIY and SVY stimulations (Supplemental Figure 7D). Cross-reactivity was also tested by analyzing the ability of SVY-expanded cells to recognize naturally processed $\mathrm{K}^{\mathrm{b}}$ SIY antigen on B16.SIY murine melanoma cells. Recognition of the naturally processed tumor antigen SIY was seen by cytokine release (Figure 3D). Therefore, heterogeneous $\mathrm{K}^{\mathrm{b} S V Y-s p e c i f i c ~} \mathrm{~T}$ cells had equal affinity for both SIY and SVY and recognized SIY-expressing tumor cells. These data highlight the differences in $\mathrm{T}$ cell populations expanded by SIY or SVY antigens, which result in subtle differences in the cross-reactive $\mathrm{T}$ cell responses. Together, this demonstrates the overall cross-reactivity of polyclonal, heterogeneous $\mathrm{T}$ cell populations that recognize 2 distinct but closely related antigens.

Bifidobacterium colonization enhances SVY-specific T cell expansion. Previously commensal Bifidobacterium was shown to promote antitumor immunity, and the bacterium associated with antitumor response was most similar to B. breve, B. longum, and B. adolescentis (99\% identity) (13). Based on the sequence homology to the cross-reactive antigen between B16.SIY and B. breve (Figure 1A), we focused our studies on the SVY antigen and examined the presence or absence of Bifidobacterium in mice obtained from Jackson or mice 


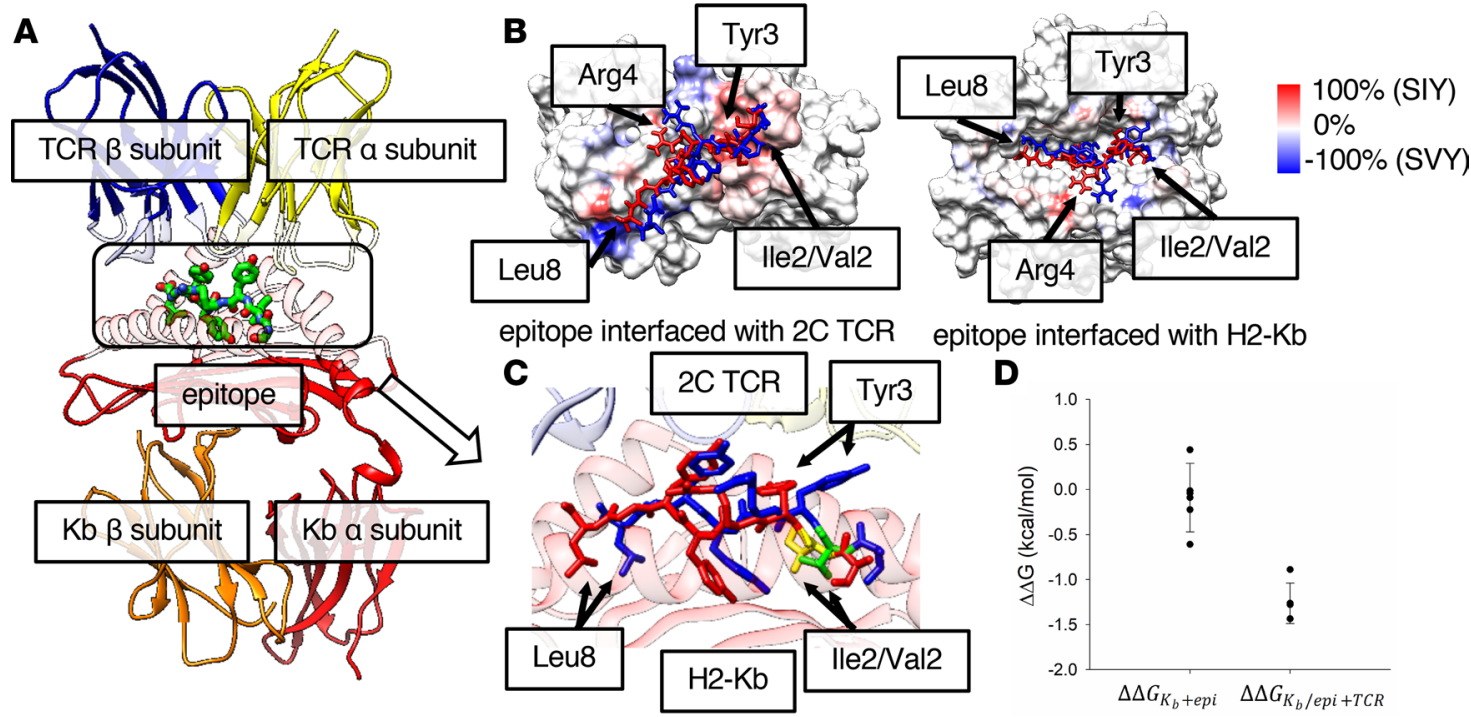

Figure 2. Modeling highlights differences in SIY and SVY binding to MHC and TCR. (A) Structure of the H2-K $/$ epitope/2C TCR complex. The $\alpha$ and $\beta$ chains of $2 \mathrm{C} \mathrm{TCR}$ and $\mathrm{H} 2-\mathrm{K}^{\mathrm{b}}$ are represented by ribbon models in yellow, blue, red, and orange, respectively, and are transparent at the epitope interface. The epitope in the box at the center with a dotted line is represented by a stick model. (B) The difference in contact frequency between the SIY and the SVY epitopes on the 2C TCR-epitope (left) and the $\mathrm{K}^{\mathrm{b}}$-epitope (right) interfaces. The SIY and the SVY epitopes are colored in red and blue, respectively. The surfaces of TCR and $\mathrm{K}^{\mathrm{b}}$ proteins are colored from blue to red, as the difference of the contact frequency is changed from $-100 \%$ (SVY) to $100 \%$ (SIY). The red color indicates that protein atoms contact the SIY epitope more often than the SVY epitope, while the blue color indicates the opposite. (C) A zoomedin image of the binding poses of the SIY (red) and the SVY (blue) epitopes, which are interfaced with $\mathrm{H} 2-\mathrm{K}^{\mathrm{b}}$ and $2 \mathrm{C}$ TCR on the top and the bottom sides of the figure. The structure of each epitope is taken from the frame at the center of the most populated cluster in the MD trajectory. Ile2 of the SIY epitope and Val2 of the SVY epitope are colored in yellow and green, respectively. The $\alpha$ and $\beta$ chains of 2C TCR and the $\alpha$ chain of $\mathrm{H} 2-\mathrm{K}^{\mathrm{b}}$ are represented by ribbon models in yellow, blue, and red, respectively. (D) The relative free energies for the binding of the epitope to $\mathrm{K}^{\mathrm{b}}\left(\Delta \Delta G_{K b+e p i}\right.$ ), and the binding of TCR to the $\mathrm{K}^{\mathrm{b}}$-epitope complex $\left(\Delta \Delta G_{K b / e p i+T C R}\right)$, between the SIY and the SVY epitopes. Each free energy value was calculated by the FEP method.

obtained from Taconic, lacking all Bifidobacterium species (Supplemental Figure 8A) (13). $\mathrm{K}^{\mathrm{b}} \mathrm{SVY}$ induced more antigen-specific T cells with a higher average MFI from Jackson than Taconic mice (Figure 4A). On average we were able to expand only approximately $8 \%$ SVY-specific T cells from Taconic mice versus $22 \%$ from Jackson mice and overall obtained about 4-fold fewer SVY-specific T cells from Taconic mice (Figure 4, B and C). A lower MFI, 109 versus 262, was also seen in cells stimulated from Taconic mice. Similarly, $\mathrm{T}$ cells from germ-free mice showed reduced frequency and total number of SVY-stimulated cells compared with Jackson mice (Supplemental Figure 8B).

Overall $\mathrm{K}^{\mathrm{b} S V Y}$-specific effector T cells from Taconic mice had a lower TCR avidity compared with $\mathrm{K}^{\mathrm{b}} \mathrm{SVY}$-specific $\mathrm{T}$ cell populations from Jackson mice (Figure 4D). However, $\mathrm{K}^{\mathrm{b}} \mathrm{SVY}$-specific $\mathrm{T}$ cells from both populations produced cytokines upon restimulation with SVY peptide-loaded RMA-S cells (Supplemental Figure 8C). The impact of Bifidobacterium colonization was also seen in analysis of SVY-specific precursor frequency. Jackson mice trended to have more $\mathrm{K}^{\mathrm{b}} \mathrm{SVY}$-specific splenocytes compared with Taconic mice (Supplemental Figure 9, A and B). Thus, B. breve exposure increases the expansion capacity of $\mathrm{K}^{\mathrm{b} S V Y}$-reactive cells, leading to higher affinity responses and more robust $\mathrm{K}^{\mathrm{b}} \mathrm{SVY} \mathrm{T}$ cell expansion.

SVY antigen is immunogenic and response is transferable via gut colonization. If SVY is indeed a cross-reactive antigen, one should be able to immunize mice and test for cross-reactive responses in vivo. Immunization of Bifidobacterium-negative Taconic mice with SVY-loaded $\mathrm{K}^{\mathrm{b}}$ complexes, as previously described (14), led to approximately $60 \%$ killing of SVY target cells in vivo and a cross-reactive killing of approximately $50 \%$ of SIY-pulsed targets in vivo (Figure $4 \mathrm{E}$ ). This was associated with an increased number of $\mathrm{K}^{\mathrm{b} S V Y}$ effector $\mathrm{T}$ cells, from $0.4 \%$ to $0.6 \%$ (Figure $4 \mathrm{~F}$ and Supplemental Figure $9 \mathrm{C}$ ). Thus, immunization with SVY elicited an in vivo response that was cross-reactive with SIY.

To investigate whether the differences seen between Jackson and Taconic mice were due to the microbiome, we studied the effect of microbiome transfer on the differences in SVY reactivity. Taconic mice cohoused with Jackson mice for a week had a significant increase in $\mathrm{K}^{\mathrm{b} S V Y}$ expansion compared with Taconic mice that were not cohoused (Figure 4G). This effect was SVY antigen specific because control $\mathrm{K}^{\mathrm{b}}$ OVA responses were not influenced by cohousing before $\mathrm{T}$ cell expansion (Figure $\left.4 \mathrm{H}\right)$. This 
A

Peptide-MHC Staining

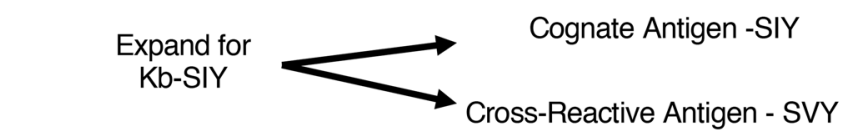

Expand for Kb-SVY

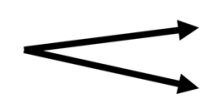

Cognate Antigen -SVY

Cross-Reactive Antigen - SIY
C

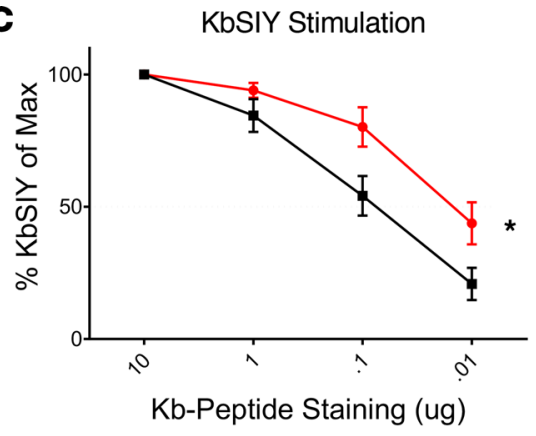

KbSVY Stimulation

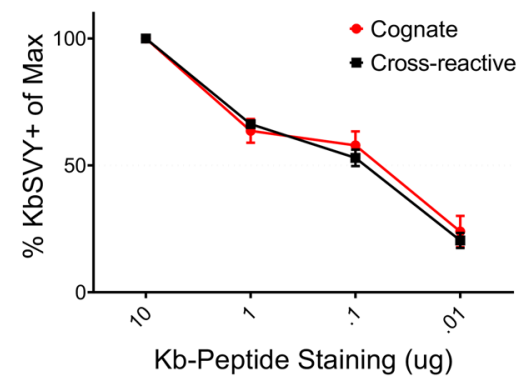

D

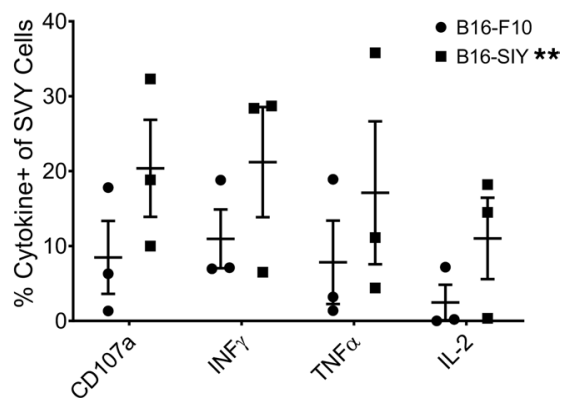

Figure 3. $K^{\mathrm{b}}$ SVY- and $K^{\mathrm{b}}$ SIY-specific $\mathbf{T}$ cells are cross-reactive. (A) Schematic for isolation, expansion, and staining of antigen-specific T cells from mouse splenocytes. Nanoparticles tethered to both peptide- $K^{\mathrm{b}}$ complexes and agonistic anti-CD28 antibodies were used as aAPCS to enrich and expand antigen-specific T cells. Expanded T cells were then stained for SIY or SVY epitope reactivity. (B) Representative flow cytometry results of $\mathrm{K}^{\mathrm{b} S I Y}$ and $\mathrm{K}^{\mathrm{b} S V Y}$ staining of antigen-specific $\mathrm{CD} 8^{+} \mathrm{T}$ cells enriched from Jackson mouse splenocytes and expanded for 7 days. Cells were gated on live $\mathrm{CD}^{+}$cells. Representative data shown from 1 of 5 separate experiments. (C) Jackson mouse splenocytes were isolated and expanded as in $\mathbf{A}$. $\mathrm{K}^{\mathrm{b}} \mathrm{SIY}$ stimulations are on top and $\mathrm{K}^{\mathrm{b}} \mathrm{SVY}$ stimulations are below. On day $7, \mathrm{CD} 8^{+} \mathrm{T}$ cells were harvested and stained with a titration of pMHC-Ig as indicated and analyzed by flow cytometry. Results are reported as percentage of peptide-MHC positivity relative to that observed at $10 \mu \mathrm{g}$ pMHC. $\mathrm{K}^{\mathrm{b} S \mathrm{Y}} \mathrm{stimu-}$ lations have a difference in cognate (red) and cross-reactive (black) binding with a $P$ value of 0.016 by 2-way ANOVA with Bonferroni's post hoc test for multiple comparisons. $\mathrm{K}^{\mathrm{b} S V Y}$ stimulation shows no difference. $P=0.016$, and $N=3$ for each antigen expansion. Data represent mean $\pm \mathrm{SEM}$. (D) $\mathrm{K}^{\mathrm{b}} \mathrm{SVY}$-reactive T cells were isolated from Jackson mouse splenocytes and expanded for 7 days as in $\mathbf{A}$. T cells were then stimulated with B16.SIY or parental B16.F10 tumor cells and measured for cytokine response by intracellular cytokine staining. $P$ value $=0.0038$ by 2-way ANOVA for B16.SIY and B16.F10 response. ${ }^{* *} P \leq 0.01 . N=3$. Data represent mean $\pm \mathrm{SEM}$.

antigen-specific $\mathrm{T}$ cell response based on microbiome colonization shows $\mathrm{CD} 8^{+} \mathrm{T}$ cells primed in vivo lead to increased expansion capabilities of SVY-specific T cells.

Jackson mice have delayed growth of B16.SIY melanoma tumors. An implication that differences in the commensal bacteria between Taconic and Jackson mice drive a cross-reactive antigen-specific targeting of B16. SIY tumors is that there should be differences in tumor growth between the 2 strains of mice. Indeed B16. SIY tumors grew more slowly in Jackson mice than in Taconic mice (Figure 5A) while B16.F10 tumors grew identically in the 2 strains (Supplemental Figure 10). Of note, initially it appeared that tumors grew slightly more rapidly in Jackson than Taconic mice (Figure 5A), but by day 10 tumors in Jackson mice were consistently smaller than in Taconic mice. By day 24, Taconic mice had an average tumor size of approximately $146 \mathrm{~mm}^{2}$, whereas tumors of Jackson mice were, on average, only $54 \mathrm{~mm}^{2}$, and individual spider plots display the slower tumor progression (Supplemental Figure 11, A and B). By day 32, all Taconic mice succumbed to disease, while $71 \%$ of the Jackson mice survived (Figure 5B). 
A

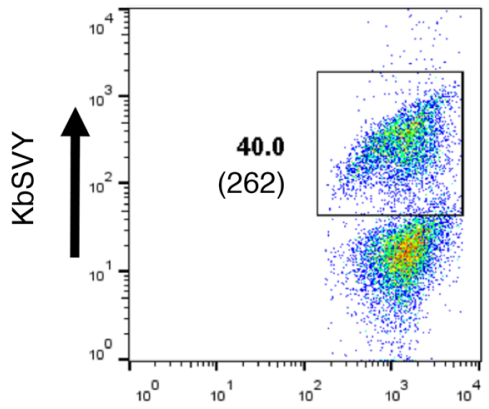

TAC

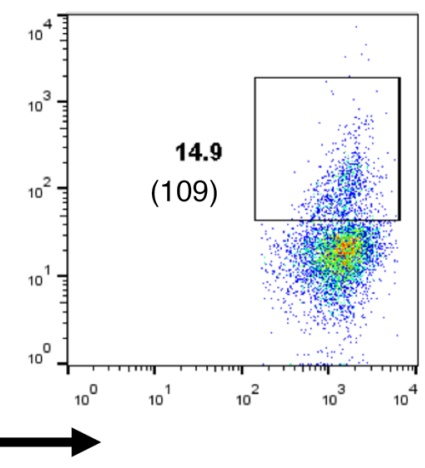

B

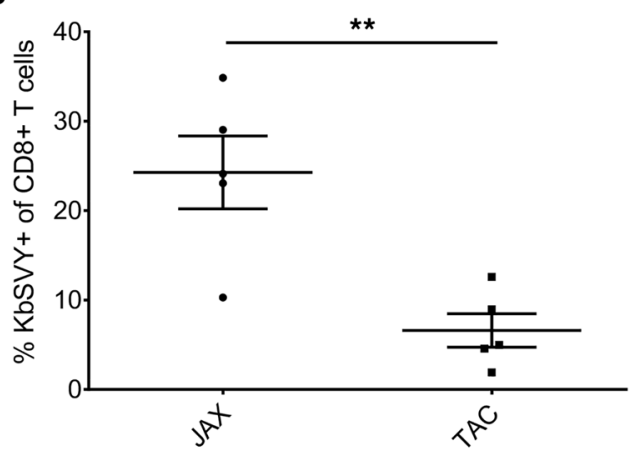

C

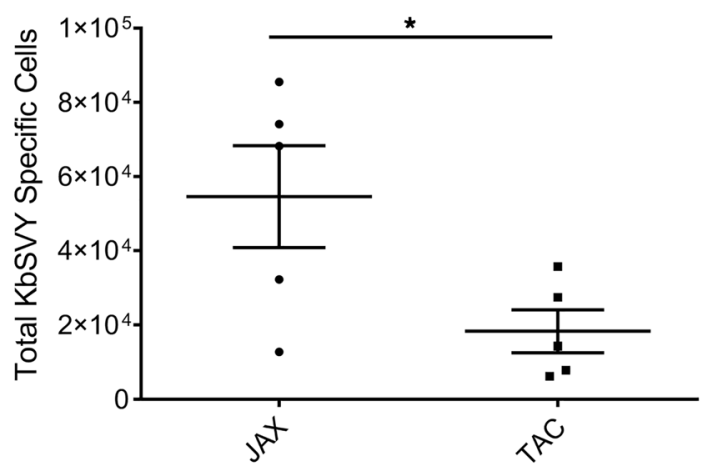

$\mathbf{F}$

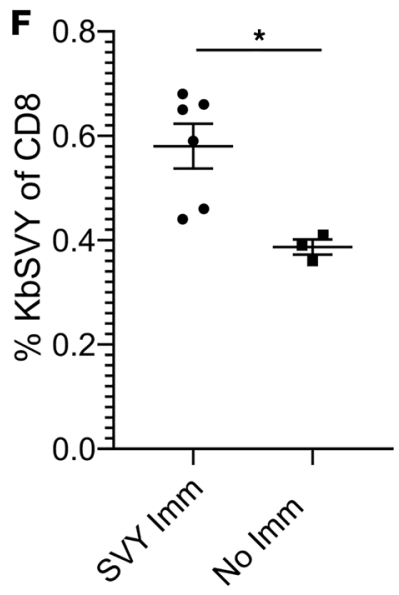

D

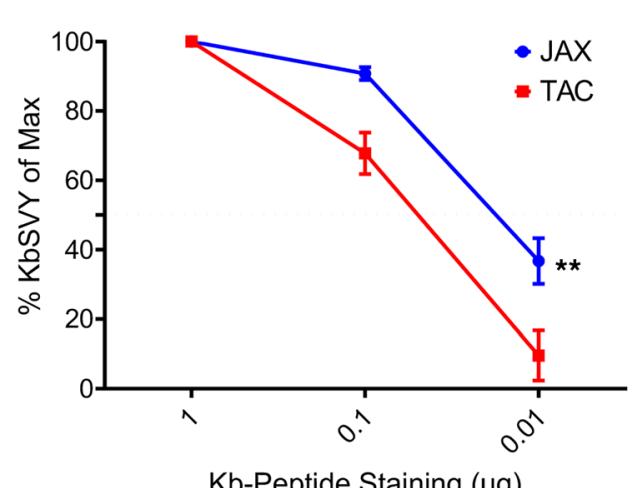

Kb-Peptide Staining (ug)
E

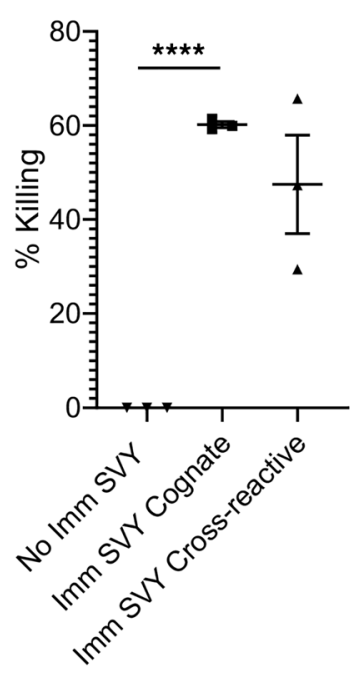

G

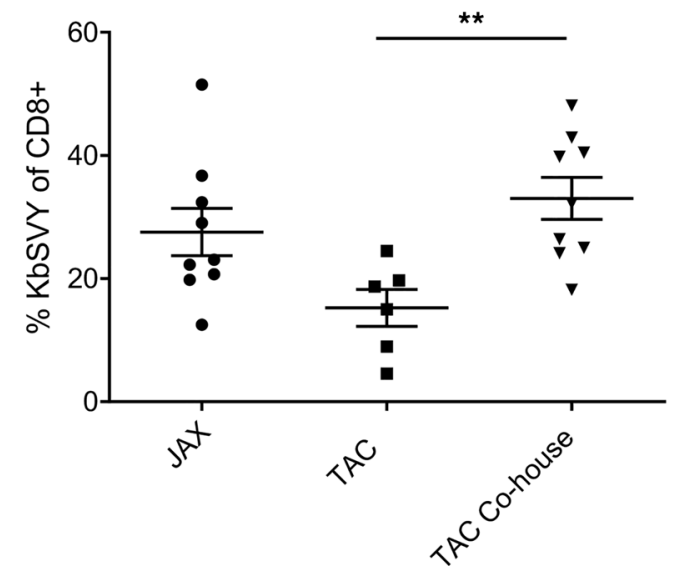

H

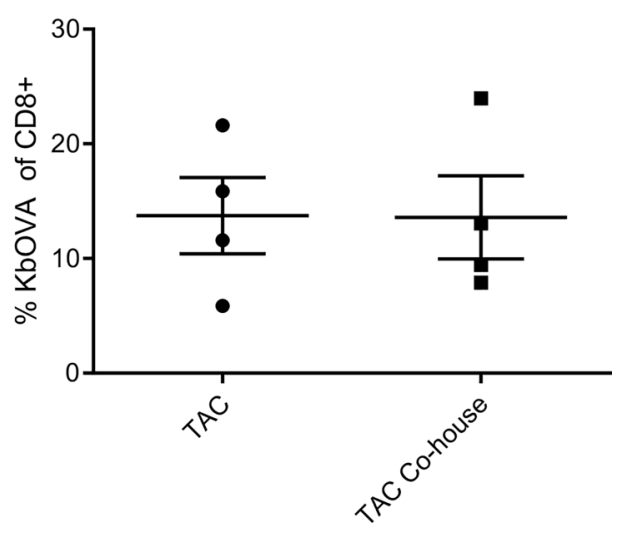

Figure 4. SVY antigen in B.breve bacteria stimulates an immunogenic $\mathbf{T}$ cell response. (A) CD8 ${ }^{+} \mathrm{T}$ cells were isolated and expanded from Jackson and Taconic splenocytes with K ${ }^{b}$ SVY aAPCS. On day 7, cells were harvested and stained for antigen specificity. Data represent 1 of 5 independent experiments. (B) CD8 ${ }^{+} \mathrm{T}$ cells were harvested and expanded as in A. Data show percentage of $\mathrm{K}^{\mathrm{b} S V Y^{+}} \mathrm{CD} 8^{+} \mathrm{T}$ cells. $P$ value $=0.0025$, measured by a 1-tailed, paired $t$ test. $N=5$. (C) Total cell numbers for CD8 ${ }^{+}$T cell enrichment and expansion with $\mathrm{K}^{\mathrm{b} S V Y}$ aAPCs. $P$ value $=0.0214$, measured by 1-tailed, paired $t$ test. $N=5$. (D) Jackson and Taconic mouse CD8 ${ }^{+} T$ cells stimulated as in A. On day 7, cells were harvested and stained with a titration of $K^{b} S V Y$. Results are reported as percentage of peptide-MHC positivity relative to that observed at $10 \mu \mathrm{g}$ pHC. Jackson (blue) and Taconic (red) peptide stains were compared at a given concentration. $P$ value $=0.0071$, measured by 2 -way ANOVA and Bonferroni's post hoc test for multiple comparisons. $N=3$. (E and $\mathbf{F}$ ) Taconic mice were immunized by intraperitoneal injection of $\alpha \mathrm{CD} 40$ on day -8 and subcutaneous injection of $K^{\mathrm{b}} \mathrm{SVY}$ on day -7 . (E) On day 0 , control or immunized mice were injected intravenously with target and nontarget autologous splenocytes mixed at a 1:1 ratio. Spleens were harvested from mice on day 1 , and the ratio of target to nontarget cells was analyzed. $P<0.0001$ by 2 -tailed, unpaired $t$ test. $N=3$ mice/group. (F) Taconic mice were sacrificed on day 1 . Spleens and draining lymph nodes were harvested and stained for $\mathrm{K}^{\mathrm{b} S V Y}$ specificity. $P=0.019$ by 2 -tailed, unpaired $t$ test. $N=6$ immunized, 3 not immunized. (C) Jackson and Taconic mice were cohoused for 7 days or separately housed with littermates. CD $8^{+}$T cells from splenocytes stimulated as in $\mathbf{A}$ and harvested and stained for antigen specificity on day 7 . Antigen specificity measured by subtracting noncognate antigen reactivity. $P$ value $=0.0014$ by 1 -tailed $t$ test. $N=5$ Taconic only; $N=8$ cohoused animals. $(\mathbf{H})$ Jackson and Taconic mice were either cohoused for 
7 days or housed with their respective littermates. CD8 ${ }^{+} T$ cells were isolated and enriched and expanded with $\mathrm{K}^{\mathrm{b}} \mathrm{OVA}$ aAPCS and harvested and stained for antigen specificity on day 7 . Antigen specificity was measured by subtracting the noncognate antigen specificity. No statistical difference seen by 1-tailed, unpaired $t$ test. $N=4$. All cells gated on live $C D 8^{+}$cells. All data represent mean \pm SEM.

We hypothesized that if differences between growth of B16.SIY tumors in Jackson versus Taconic mice were driven by antigen-specific recognition of the SIY neoantigen, we should see evidence for pressure on SIY antigen in tumors from Jackson mice. Tumors were collected from individual mice and analyzed for GFP expression, which is encoded by the same vector as SIY in the B16.SIY tumors. This approach has been used for a surrogate marker of anti-SIY antigen-specific T cell responses (15). B16.SIY tumors in Jackson mice had a significant loss of GFP expression, from 50\% in Taconic mice tumors to $10 \%$ in Jackson mice tumors (Figure 5C and Supplemental Figure 11C).

Tumor-infiltrating T cells in B16.SIY tumors show cross-reactivity for $K^{b} S I Y$ and $K^{b} S V Y$ in Jackson mice tumors. Jackson mice tumor-infiltrating $\mathrm{T}$ lymphocytes (TILs) were stained for $\mathrm{K}^{\mathrm{b}} \mathrm{SIY}$ specificity or cross-reactivity for $\mathrm{K}^{\mathrm{b} S V Y}$ and showed substantial staining over background $\mathrm{K}^{\mathrm{b}} \mathrm{OVA}$, which is not expected to be present in the B16.SIY tumor (Figure 5D, Supplemental Figure 11D). Notably, when TCR $\beta$ chain sequencing was performed on TILs derived from B16.SIY tumors, we found that $22 \%$ of TIL clones were identical to those detected independently in splenocyte populations sorted and expanded by $\mathrm{K}^{\mathrm{b}} \mathrm{SVY}$. Overlapping TIL clones consisted of 9 of the $198 \mathrm{~K}^{\mathrm{b} S V Y}$-reactive sequences identified by in vitro enrichment and expansion of healthy mouse splenocytes $\left(P=3.42 \times 10^{-30}\right.$ for enrichment of SVY-reactive clones in the TIL population by the hypergeometric test). Taken together, Jackson mice have a beneficial antitumor response that is antigen driven and have higher selective pressure against the tumor antigen, and $\mathrm{K}^{\mathrm{b}} \mathrm{SVY}$ cross-reactive $\mathrm{T}$ cells can be detected in the tumor, all highlighting the beneficial cross-reactive $\mathrm{T}$ cell population that is present in the Jackson mice.

Bifidobacterium alters the composition of the SVY-responsive TCR repertoire. The impact of $B$. breve colonization on $\mathrm{T}$ cell specificity was also studied by analyzing TCR repertoires expanded by $\mathrm{K}^{\mathrm{b}} \mathrm{SIY}$ or $\mathrm{K}^{\mathrm{b}} \mathrm{SVY}$ from B. breve-colonized (Jackson) mice and those lacking B. breve (Taconic). $\mathrm{K}^{\mathrm{b}} \mathrm{SIY}$ - and $\mathrm{K}^{\mathrm{b} S V Y-s p e c i f i c ~} \mathrm{~T}$ cells from Jackson and Taconic mice were stimulated and sorted based on $\mathrm{K}^{\mathrm{b}} \mathrm{SIY}$ or $\mathrm{K}^{\mathrm{b}} \mathrm{SVY}$ staining (see Figure 6A schematic). The $8 \mathrm{~T}$ cell populations were analyzed by TCR $\beta$ chain sequencing to determine how $B$. breve shapes $\mathrm{K}^{\mathrm{b}} \mathrm{SVY}$ TCR repertoires.

All 4 expanded TCR repertoires showed significant overlap when stained with cognate or cross-reactive $\mathrm{K}^{\mathrm{b}}$-peptide dimer (Figure $6 \mathrm{~B}$ ). Independent of B. breve colonization, we found many overlapping clones when comparing the cognate and cross-reactive sorted populations. As seen in the heatmap analysis (Supplemental Figure 12, A and B), the overlapping pairs were in distinct groups with little overlap with other repertoires and led to a selection of 4 distinct TCR populations of SIY and SVY cross-reactive clones (Figure 6B). Within Jackson mice $\mathrm{T}$ cell repertoires expanded by $\mathrm{K}^{\mathrm{b}} \mathrm{SIY}$, the $\mathrm{K}^{\mathrm{b}} \mathrm{SIY}$-sorted and $\mathrm{K}^{\mathrm{b}}$ SVY-sorted populations shared 47 identical clones comprising $79.9 \%$ of the response (Figure $6 \mathrm{~B}$ ). These populations also shared the same $\mathrm{V}$ and $\mathrm{J}$ allele expression based on heatmap analysis (Supplemental Figure 13, A and B). Within Jackson mice $\mathrm{T}$ cell repertoires expanded by $\mathrm{K}^{\mathrm{b}} \mathrm{SVY}$, the $\mathrm{K}^{\mathrm{b}} \mathrm{SVY}$-sorted and $\mathrm{K}^{\mathrm{b}} \mathrm{SIY}$-sorted populations shared 93 unique clones comprising $95.6 \%$ of the response (Figure $6 \mathrm{~B}$ ). In Taconic mice, $\mathrm{T}$ cell populations expanded with $\mathrm{K}^{\mathrm{b}} \mathrm{SIY}$ and sorted on $\mathrm{K}^{\mathrm{b}} \mathrm{SIY}$ or $\mathrm{K}^{\mathrm{b}} \mathrm{SVY}$ had 45 overlapping clones that made up $85.9 \%$ of the response, while the $\mathrm{T}$ cells expanded with $\mathrm{K}^{\mathrm{b}} \mathrm{SVY}$ and sorted on $\mathrm{K}^{\mathrm{b}} \mathrm{SVY}$ or $\mathrm{K}^{\mathrm{b}} \mathrm{SIY}$ had 49 shared clones comprising $77.1 \%$ of the response. Thus, identical $\mathrm{T}$ cell clones from cognate and cross-reactive staining of expanded cells showed that $\mathrm{T}$ cells are cross-reactive for both antigens, regardless of the commensal bacteria colonization.

The relationship between TCR repertoires was also examined using ImmunoMap, a TCR homology program we recently developed (16). ImmunoMap characterizes the overall homology patterns between different $\mathrm{T}$ cell repertoires based on homologous complementarity-determining region 3 (CDR3) sequences. In this fashion multiple repertoires can be overlaid to compare homology among various TCR repertoires (Supplemental Table 3). The effect of B. breve on TCR repertoire composition can be seen when comparing the overlap of SIY or SVY stimulation in Jackson and Taconic mice (Figure 6, C and D). B. breve-negative mice (Taconic) cells stimulated with $\mathrm{K}^{\mathrm{b}} \mathrm{SVY}$ reactivity have only $51.1 \%$ homology to the $\mathrm{K}^{\mathrm{b} S V Y}$-stimulated repertoire from B. breve-positive (Jackson) mice (Figure 6C, note highlighted boxes; Figure 6D; and Supplemental Figure 12B). In contrast, the $\mathrm{K}^{\mathrm{b}} \mathrm{SIY}$ TCR repertoire had a significantly overlapping response between Jackson and Taconic stimulations, with $71 \%$ overlap between both repertoires, which highlights that $B$. breve colonization has a greater effect on the composition of the $\mathrm{K}^{\mathrm{b}} \mathrm{SVY}$-stimulated TCR repertoire than on that of the $\mathrm{K}^{\mathrm{b}} \mathrm{SIY}$-stimulated repertoire (Figure $6 \mathrm{D}$ and Supplemental Figure 12). 
A

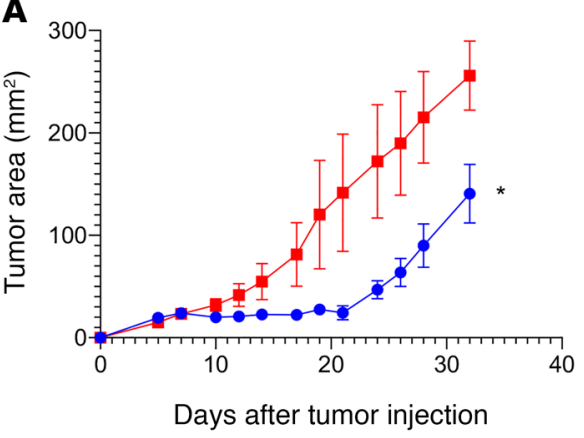

C

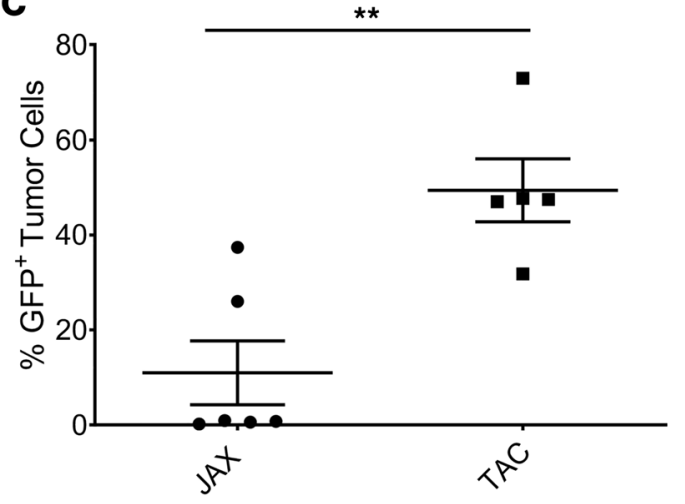

B

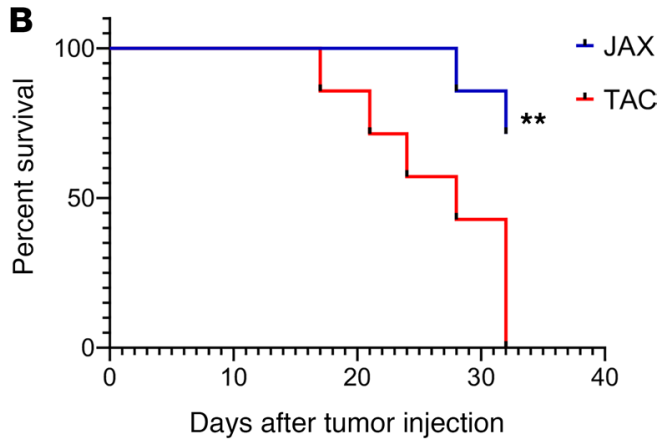

D

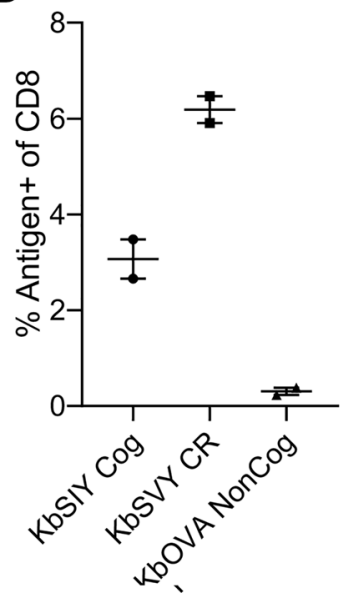

Figure 5. Jackson mice have an increased antitumor response and antigen-selective pressure on the tumor. (A) Jackson and Taconic mice were injected with $2 \times 10^{6}$ B16.SIY cells subcutaneously on day 0 . Tumor growth curves show Jackson mice have significantly delayed tumor growth as compared with Taconic mice. $P$ value $=0.0455$. Significance was measured by 2-way ANOVA with Bonferroni's post hoc test for multiple comparisons. $N=7 /$ group. (B) Additionally, Jackson mice had significantly increased survival compared with the Taconic mice. $P=0.0065$. Significance was measured by the log-rank test (Mantel-Cox test). $N=7 /$ group. (C) Jackson and Taconic mice were injected with $2 \times 10^{6}$ B16.SIY cells subcutaneously on day 0 . Tumors were harvested at an approximate size of $200-250 \mathrm{~mm}^{2}$ and analyzed by flow cytometry for GFP expression, as SIY and GFP are expressed as a fusion protein in the B16.SIY cell line (15). Jackson have a significantly lower GFP expression $(P$ value $=0.0030)$ by unpaired, 2-tailed $t$ test $(n=5$ TAC, and $n=6$ JAX). (D) Jackson and Taconic mice were injected with $2 \times 10^{6}$ B16.SIY cells subcutaneously on day 0 . Tumor-infiltrating lymphocytes were harvested on day 24 and analyzed by flow cytometry for CD8 ${ }^{+} T$ cell specificity, gated on live $\mathrm{CD} 8^{+}$T cells. $N=2 /$ group.

Principal component analysis (PCA) was used to compare the TCR clones based on homology of dominant motifs identified from repertoires (16). Although individual repertoires all shared similar diversity metrics (Sup-

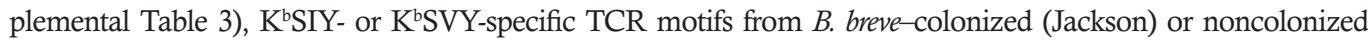
(Taconic) mice revealed 4 distinct pairings, each driven by the supplier and the epitope used for expansion; however, the separation within and between pairs was qualitatively greater for Taconic mice than for Jackson mice (Figure 6E). Taken together, TCR repertoire sequencing indicates that the majority of SIY- or SVY-stimulated T cell clones are cross-reactive with both epitopes and that $B$. breve colonization is associated with a significantly greater shift in the clonal composition of the SVY-stimulated repertoire than in that of the SIY-stimulated repertoire.

The effect of Bifidobacteria stimulation on the TCR repertoire was also analyzed on T cells stimulated in vitro with heat-killed Bifidobacteria. After bacterial stimulation, TCR repertoire analysis of the expanded $\mathrm{T}$ cells identified 255 unique clones that overlapped with Jackson $\mathrm{K}^{\mathrm{b}} \mathrm{SIY}$ - or $\mathrm{K}^{\mathrm{b}} \mathrm{SVY}$-stimulated and sorted repertoires by a Hamming distance of 1 . The TCR clones similar to SIY- or SVY-peptide-reactive TCR repertoires were at a significantly higher productive frequency compared with clones present in nonBifidobacteria-stimulated controls (Figure 6F). Thus, similar to our in vivo findings, in vitro stimulation with Bifidobacteria expanded a $\mathrm{K}^{\mathrm{b}}$ SIY-reactive TCR repertoire that could have antitumor activity. 
A

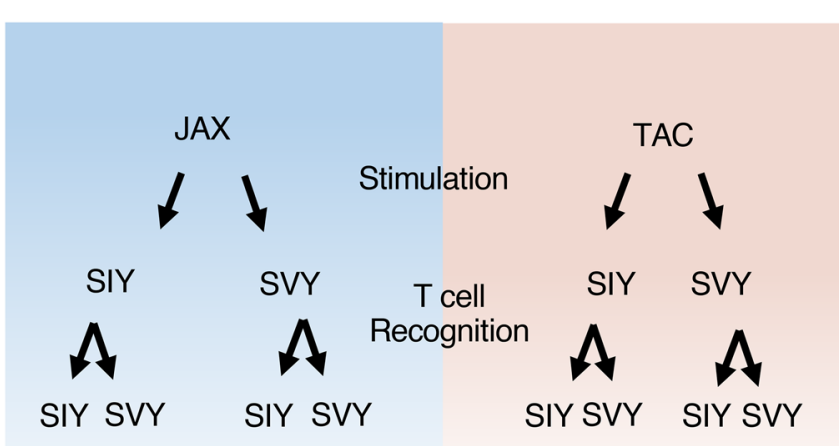

C
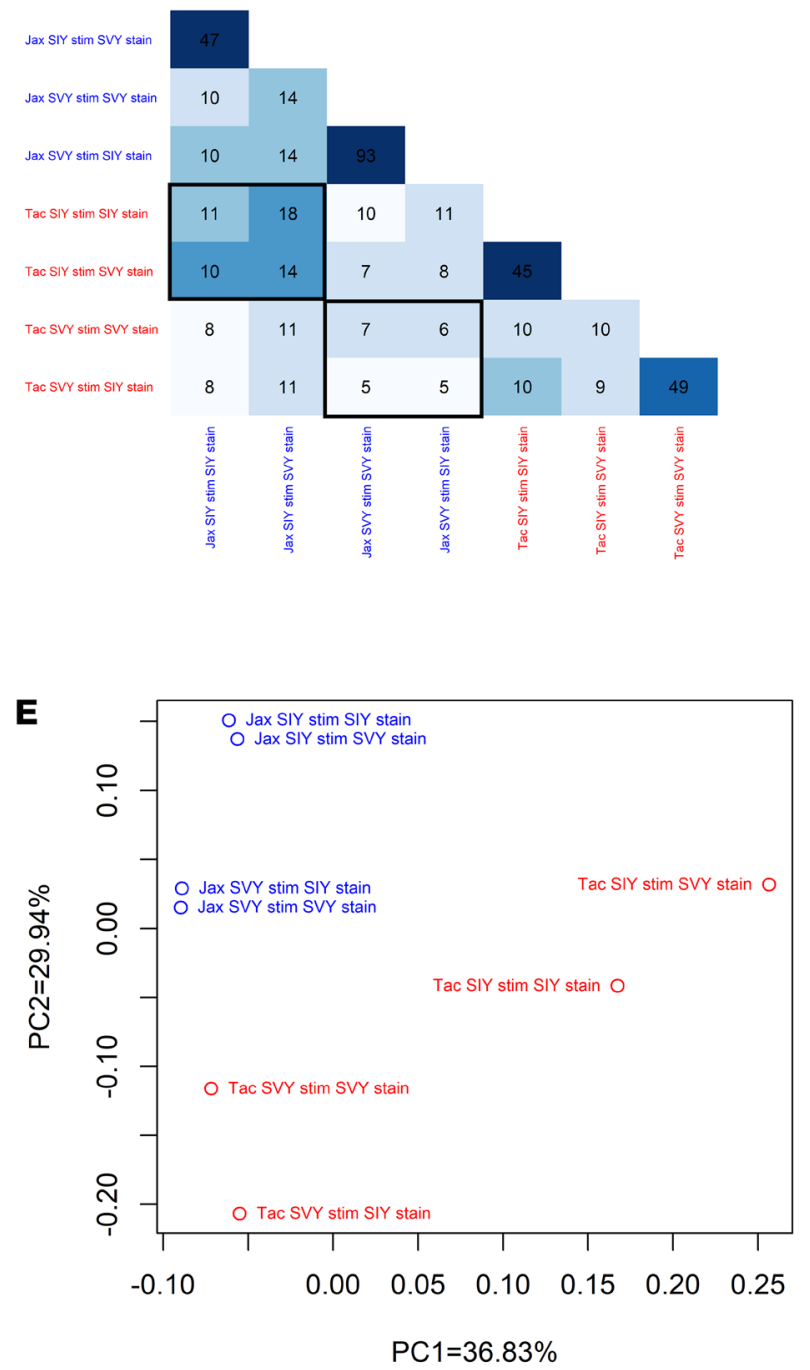

B

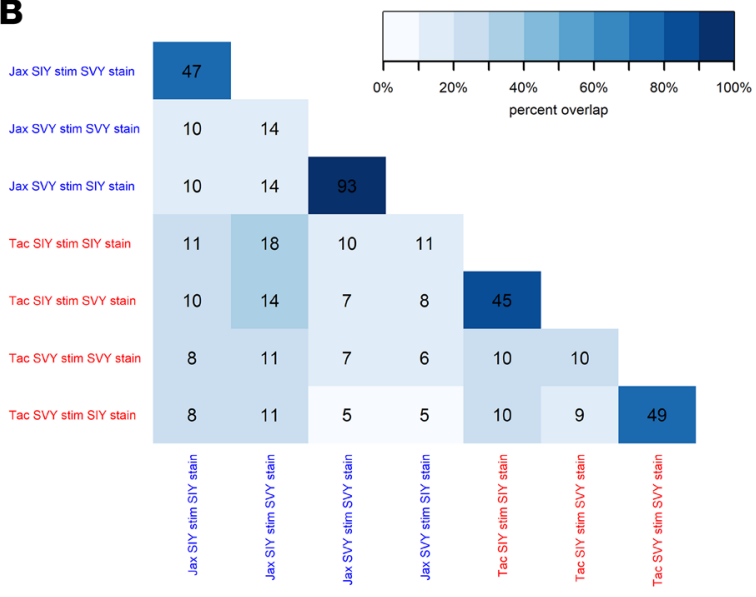

D

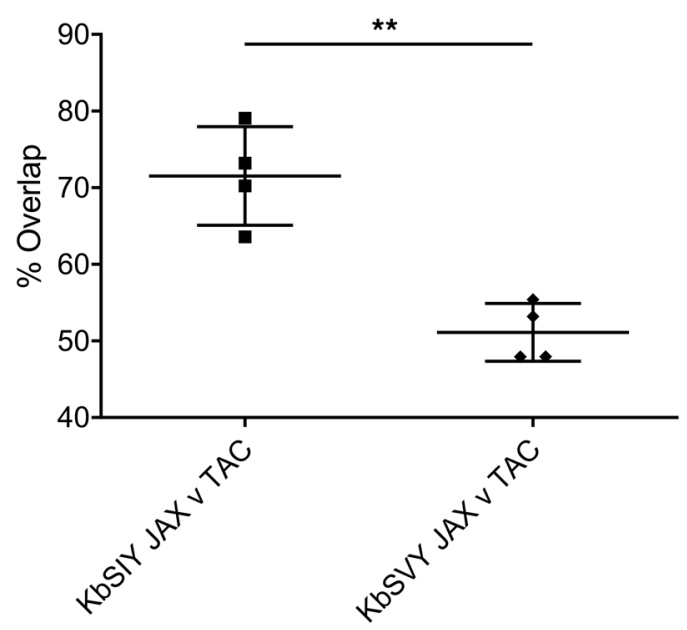

$\mathbf{F}$

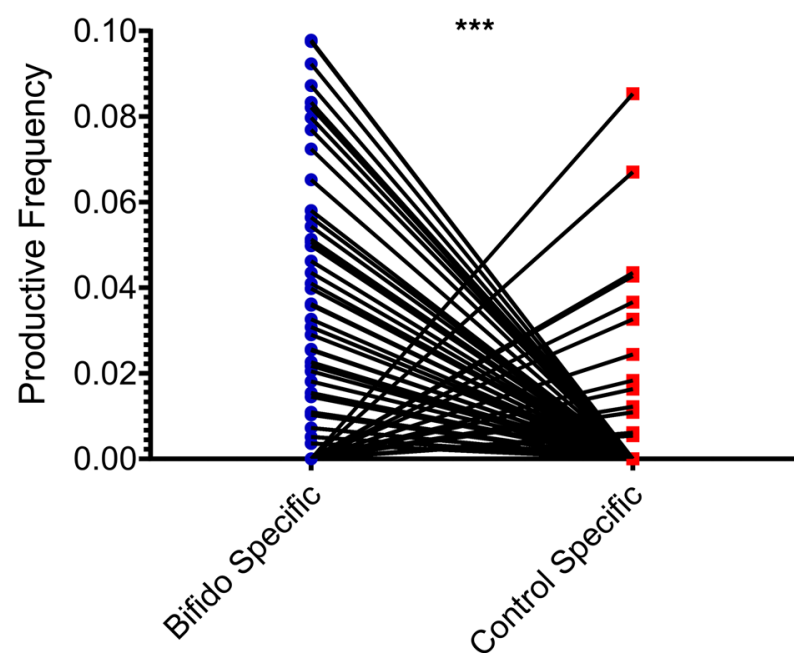

Figure 6. B. breve colonization shapes the K' ${ }^{\mathrm{b}} \mathrm{SVY}$ TCR repertoire. (A) Schematic setup of TCR repertoire analysis. CD8 ${ }^{+} \mathrm{T}$ cells from Jackson and Taconic mice were stimulated with $\mathrm{K}^{\mathrm{b}} \mathrm{SIY}$ or $\mathrm{K}^{\mathrm{b}} \mathrm{SIY}$ aAPCs. On day 7, cells from each stimulation were stained and sorted by antigen reactivity ( $\mathrm{K}^{\mathrm{b} S I Y}$ or $\mathrm{K}^{\mathrm{b} S V Y}$ ) and processed for TCR $\beta$ chain deep sequencing. (B) Exact TCR clone overlap between all repertoires. Numbers indicate the number of unique overlapping clones between 2 TCR repertoires, and the color scale indicates the percentage contribution of the overlapping sequences to the total combined repertoire for each pair. (C) TCR clone overlap between all repertoires based on TCR homology using ImmunoMap algorithm. Numbers indicate the number of homologous clusters shared between 2 TCR repertoires, and the color scale indicates the percentage contribution of shared clusters to the total combined repertoire for each pair. (D) Overlap between Jackson and Taconic mice of T cell repertoires stimulated by SIY or SVY based on the frequency of homologous TCR clones and overlap between SIY and SVY stimulations for Jackson or Taconic mice groups. Data included the cognate and cross-reactive sorted 
populations, as indicated by the black boxes in C. $P$ value $=0.0016$ by 2-tailed $t$ test. $N=4$. Data represent mean \pm SEM. (E) PCA of homology-based TCR clusters. TCR repertoires were analyzed by CDR3 sequence homology and separated into dominant motifs. (F) TCR $\beta$ chain deep sequencing was performed on Jackson mouse splenocytes incubated with or without heat-killed Bifidobacterium. TCR clones with a Hamming distance of 1 were defined as homologous to clones from aAPC-expanded K ${ }^{\mathrm{b} S I Y}$ - or K ${ }^{\mathrm{b} S V Y-s p e c i f i c ~ T ~ c e l l s . ~ P r o d u c t i v e ~ f r e q u e n c y ~ o f ~ t h e s e ~ h o m o l o g o u s ~ c l o n e s ~ f r o m ~ B i f i d o b a c t e r i u m-s t i m u l a t e d ~}$ and unstimulated cells is shown. $P$ value $=0.0001$ by 2 -tailed $t$ test.

$K^{b} S V Y$-reactive $T$ cells inhibit growth of established SIY-expressing tumors in vivo. One critical question is whether $\mathrm{K}^{\mathrm{b}} \mathrm{SVY}$-reactive $\mathrm{T}$ cells cross-react with the SIY epitope in vivo and mediate an effective antitumor response. This was studied using $\mathrm{K}^{\mathrm{b}} \mathrm{SVY}$ effector $\mathrm{T}$ cells from $B$. breve-positive mice (Jackson) for ACT. Effector T cell populations were generated in vitro and adoptively transferred into B16.SIY tumor-bearing mice. $\mathrm{K}^{\mathrm{b}}$ SVY-expanded T cells inhibited B16.SIY tumor growth and extended survival in tumor-bearing mice. By day 22, untreated mice had an average tumor size of approximately $89.87 \mathrm{~mm}^{2}$, whereas SVY ACT-treated mice were, on average, only $22.37 \mathrm{~mm}^{2}$ (Figure 7A and Supplemental Figure 14A). When we analyzed survival data, $60 \%$ of $\mathrm{K}^{\mathrm{b} S V Y} \mathrm{ACT}$-treated mice survived past 41 days while no control mice survived that long (Figure 7A).

ACT-treated mice, which eventually developed resistant tumors, were analyzed for antigen loss. Tumors were isolated at approximately $135 \mathrm{~mm}^{2}$ and measured for GFP intensity as a marker of SIY expression (17). The resistant tumors had a decrease in GFP expression compared with tumors isolated from an untreated mouse (Figure $7 \mathrm{~B}$ ), demonstrating that $\mathrm{K}^{\mathrm{b}}$ SVY-specific cells placed selective pressure on B16.SIY tumors, resulting in loss of antigen expression. As expected, $\mathrm{K}^{\mathrm{b}} \mathrm{SIY}$-expanded $\mathrm{T}$ cells also delayed tumor progression in tumor-bearing mice and extended survival (Figure 7C), showing that the repertoire present in $\mathrm{K}^{\mathrm{b}} \mathrm{SIY}$-specific $\mathrm{T}$ cells also has antitumor activity. Thus polyclonal, cross-reactive $\mathrm{T}$ cell populations expanded with $\mathrm{K}^{\mathrm{b}} \mathrm{SVY}$ decreased tumor growth and increased survival, demonstrating the potential benefits of commensal bacteria-responsive $\mathrm{T}$ cell cross-reactivity on antitumor immunity.

\section{Discussion}

Understanding the role of antigen mimicry leading to $\mathrm{T}$ cell-mediated antitumor responses is essential in understanding how microbiota can lead to antitumor effects. B. breve contains a peptide with homology to the SIY epitope, which influences anti-B16.SIY melanoma-specific responses. Use of $B$. breve as a source of homologous antigen does not limit the possibility of other sources of antigen, although throughout the paper the $B$. breve antigen sequence shows robust cross-reactivity and ability to induce the antitumor $\mathrm{T}$ cell response both in vitro and in vivo. Looking closely, the isoleucine substitution to valine at the second position did not affect peptide-MHC binding; however, the substitution appears to shift the orientation of more distant amino acids in the binding pocket, leading to altered TCR recognition. Although differences in binding with the 2C transgenic TCR illustrated that an individual TCR can display somewhat lower affinity for $\mathrm{K}^{\mathrm{b}} \mathrm{SVY}$ than $\mathrm{K}^{\mathrm{b}} \mathrm{SIY}$, endogenous murine polyclonal SIY and SVY T cell responses exhibit striking cross-reactivity. On closer examination, TCR $\beta$ sequencing reveals the same clones can bind to both the SIY and SVY antigens after a single stimulation. The endogenous $\mathrm{K}^{\mathrm{b}} \mathrm{SIY}$ response is cross-reactive and recognizes both antigens, SIY and SVY, albeit with a lower affinity and less robust functional response to the SVY antigen. In contrast, $\mathrm{K}^{\mathrm{b}} \mathrm{SVY}$-reactive $\mathrm{T}$ cells recognize both peptide antigens with similar affinity as well as functional ability, as assessed by their ability to release cytokines in response to tumor cells. This difference in cross-reactivity relative to the initial $\mathrm{T}$ cell stimulation suggests that C57BL/ 6 mice possess a heterogeneous SIY/SVY T cell population that can be stimulated by $B$. breve and that the polyclonal population contains a range of affinities for SIY and SVY based on ability to bind to the slightly altered amino acid orientation in the MHC pocket.

One of the most interesting findings is that the gut microbiota primes an SVY-reactive $\mathrm{T}$ cell response. Although the mechanism of $\mathrm{T}$ cell activation and selection by components of the gut microbiota is not understood, analysis of mice with and without Bifidobacterium indicates that in vivo priming occurs and boosts the ability to expand SVY-reactive T cells. The SVY antigen is immunogenic and can induce effector T cells that are cross-reactive in vivo with the SIY antigen. In addition, the antigen-specific T cell response is transferable, as demonstrated by cohousing experiments. Comparing the TCR repertoires between colonized and noncolonized animals revealed SVY-reactive T cells that express TCRs with little or no overlap between the $B$. breve-naive (Taconic) and B. breve-experienced (Jackson) mouse repertoires. An altered SVY-expandable TCR repertoire developed in the colonized mice, while the SIY-expandable populations showed markedly 
A
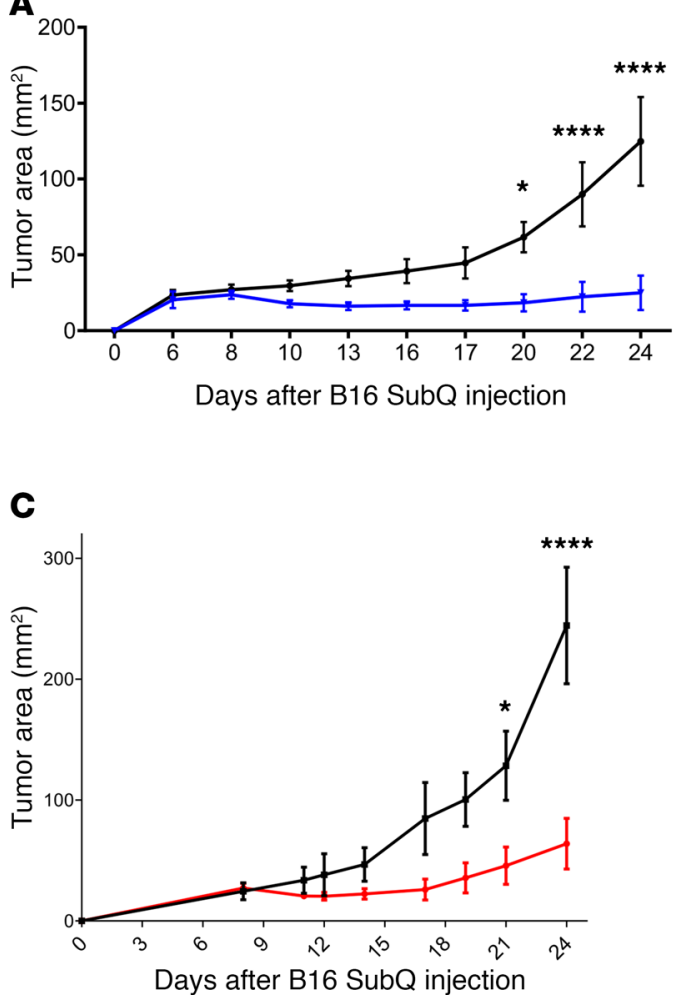

B

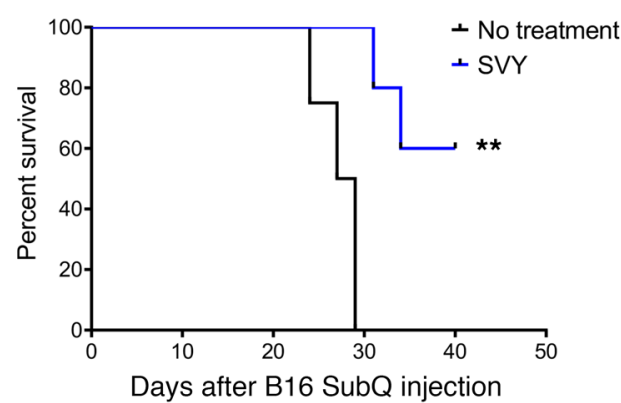

Days after B16 SubQ injection

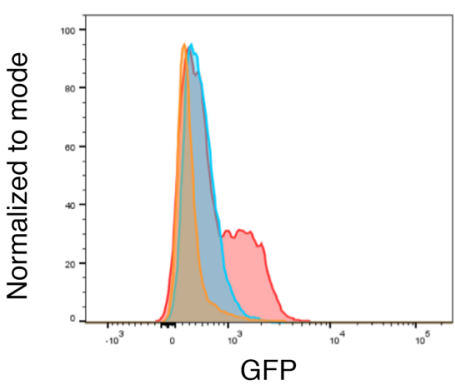

Untreated tumor SVY ACT tumor SVY ACT tumor

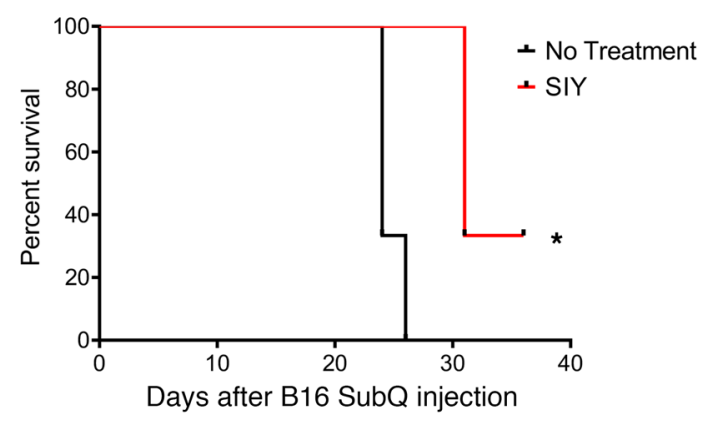

Figure 7. Commensal bacteria epitope cross-reactivity mediates an antitumor response. (A) Jackson mice ( $n=5$ SVY ACT; $n=4$ no treatment) were injected with $2 \times 10^{6} \mathrm{~B} 16 . \mathrm{SIY}$ cells subcutaneously on day 0 . CD8 ${ }^{+} \mathrm{T}$ cells were harvested from spleens of independent Jackson mice and stimulated with $\mathrm{K}^{\mathrm{b}} \mathrm{SVY} / \mathrm{anti}-\mathrm{CD} 28$ nanoparticles. On day $8,1.3 \times 10^{5}$ of the resultant $\mathrm{K}^{\mathrm{b}} \mathrm{SVY}$-specific CD8 ${ }^{+} \mathrm{T}$ cells were injected intravenously into the tumor-bearing mice. Tumor growth curves show SVY ACT treatment significantly delayed tumor growth as compared with no treatment. $P$ value $=0.0136$, measured by 2 -way ANOVA with Bonferroni's post hoc test for multiple comparisons. Additionally, K' ${ }^{\mathrm{b}} \mathrm{SV}$ ACT significantly increased survival compared with the no treatment group. Significance was measured by the log-rank test. $P$ value $=0.0015$. (B) Jackson mice were injected with $2 \times 10^{6}$ B16.SIY cells subcutaneously on day 0 and treated on day 8 with $1.3 \times 10^{5} \mathrm{~K}^{\mathrm{b} S V Y}$-specific CD8 ${ }^{+} \mathrm{T}$ cells via intravenous injection. Tumors were harvested on day 24 and analyzed by flow cytometry for GFP expression. Orange and blue indicate SVY T cell-treated mice and red indicates untreated mice. (C). Jackson mice ( $n=3$ SIY ACT, and $n$ $=3$ no treatment) were injected with $2 \times 10^{6}$ B16.SIY cells subcutaneously on day 0 . CD8 ${ }^{+}$T cells were harvested from spleens of independent Jackson mice and stimulated with $\mathrm{K}^{\mathrm{b}} \mathrm{SIY} / \mathrm{anti}-\mathrm{CD} 28$ nanoparticles. On day $8,1.3 \times 10^{5}$ of the resultant SIY-reactive T cells were injected intravenously into tumor-bearing mice. Tumor growth curves show SIY ACT treatment significantly delayed tumor growth as compared with no treatment. Significance was measured by 2-way ANOVA with Bonferroni's post hoc test for multiple comparisons $(P<0.001)$. ACT also significantly extended survival. Significance was measured by the log-rank test. $P$ value $=0.024 . N=3 /$ group.

greater homology between Taconic and Jackson mice. The SVY-stimulated TCR repertoires from B. brevecolonized (Jackson) mice contained homologous TCR clones that are shared with the Jackson $\mathrm{K}^{\mathrm{b}} \mathrm{SIY}$ stimulation. These findings suggest that differences in B16.SIY and B16.F10 tumor growth between Taconic and Jackson mice may be driven in part by a cross-reactive, SVY-primed subset of the TCR repertoire. In addition, stimulating $\mathrm{T}$ cells directly with $B$. breve expands $\mathrm{T}$ cell clones that are similar to the Jackson $\mathrm{K}^{\mathrm{b}} \mathrm{SIY}$ or $\mathrm{K}^{\mathrm{b}} \mathrm{SVY}$ $\mathrm{T}$ cell populations. These data highlight how commensal bacterial antigens may affect the host immune repertoire landscape through antigen mimicry. An important characteristic of the SVY-reactive T cells was their ability to kill SIY-expressing tumors in vivo. Treatment of B16.SIY tumor-bearing animals with adoptively transferred $\mathrm{K}^{\mathrm{b}} \mathrm{SVY}$-reactive $\mathrm{T}$ cells showed cross-reactive antitumor activity of an endogenous, polyclonal commensal bacteria antigen-stimulated $\mathrm{T}$ cell population.

Importantly, our study illustrates that antigen mimicry from gut microbes influences $\mathrm{T}$ cell immunity and helps generate a cross-reactive antitumor response. Current work identifying beneficial bacteria from responders to immunotherapy as well the benefits of fecal transplant in patients has yet to isolate a clear mechanism of action; antigen mimicry may play a role in patient responses. Here we confirmed that antigen homology between Bifidobacterium and the murine melanoma SIY "neoantigen" stimulates cross-reactive $\mathrm{T}$ cells. Furthermore, the presence of $B$. breve in the microbiome boosts the $\mathrm{K}^{\mathrm{b}} \mathrm{SVY} \mathrm{T}$ cell population,

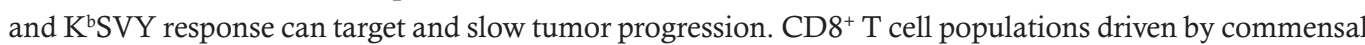
bacteria stimulation have shown functional responses $(7,18-21)$, and we now reveal how changing the 
microbiome can alter cross-reactive $\mathrm{T}$ cell responses, which highlights the importance of the microbiome for $\mathrm{CD}^{+} \mathrm{T}$ cell responses. Although the overall diversity of the microbiome has been linked to positive clinical responses in patients, identifying homologous antigens found in commensal bacteria relevant to specific patients' tumors will require more extensive studies with potentially a more robust, larger array of antigens to stimulate the $\mathrm{T}$ cell response $(7,22,23)$. With this mechanism in mind, we believe that a rigorous panspecies genomic analysis of commensal organisms would aid a more precise understanding of the effects of microbiota on antitumor immunotherapy. Tumor neoantigen homology to pathogen or commensal bacteria may provide an effective way to isolate unique $\mathrm{T}$ cell populations that can be stimulated by both gut microbes and tumor cells and to generate robust antitumor $\mathrm{T}$ cell responses.

\section{Methods}

Mice. C57BL/6 mice were purchased from The Jackson Laboratory (Bar Harbor, Maine, USA) and Taconic Biosciences (Germantown, New York, USA). 2C TCR transgenic mice were a gift from the Gajewski laboratory (University of Chicago, Chicago, Illinois, USA) and bred as heterozygotes on the C57BL/6 background. Male and female mice, 8-12 weeks old, were used and maintained according to Johns Hopkins University's (JHU) Institutional Review Board.

Cell lines. RMA-S cells were a gift from the Edidin laboratory (JHU, Baltimore, Maryland, USA). B16. F10 cells were a gift from the Mackensen laboratory (Alfred Wegener Institute, Bremerhaven, Germany). B16.SIY cells were a gift from the Drake laboratory (JHU, Baltimore, Maryland, USA).

Peptide stabilization assay. RMA-S cells were held at $25^{\circ} \mathrm{C}$ overnight and pulsed with peptide (GenScript) for 2 hours, then incubated at $37^{\circ} \mathrm{C}$ for 2 hours. Cells were stained with anti- $\mathrm{K}^{\mathrm{b}}(\mathrm{M} 1 / 42$, BioLegend) and run on flow cytometry.

2C functional analysis. 2C splenocytes were stimulated with peptide for 5 days, harvested and restimulated with RMA-S cells, and peptide pulsed overnight. T cells and RMA-S cells were incubated (1:1) with GolgiStop (554724, BD Biosciences), GolgiPlug (555029, BD Biosciences), and CD107a (FITC, 1D4B, BD Pharmingen). After 6 hours, cells were stained with viability stain (L23101, Thermo Fisher Scientific) and anti-CD8 ${ }^{+}$(PerCP, 53-6.7, BioLegend). Cells were fixed and permeabilized (Fixation/Permeabilization Solution Kit 554714, BD Biosciences) and stained for IFN- $\gamma$ (PE, XMG1.2, BD Pharmingen), TNF- $\alpha$ (PE-Cy7, MP6-XT22, BioLegend), and IL-2 (APC, JES6-5H4, BD Pharmingen).

Competitive binding assay. 2C T cells were stimulated with SIY peptide for 5 days and stained with fluorescent peptide-MHC multimer/(in-house) for 1 hour. Afterward, saturating amounts of an unconjugated competitor anti-mouse 2C TCR (1B2, Bio X Cell) was added and measured by flow cytometry over time.

Bifidobacteria quantification. Fecal DNA samples were isolated from Jackson and Taconic mice (R2002, Zymo Research). Bifidobacteria colonization relative to total eubacteria was quantified by multiplexed quantitative real-time PCR analysis of microbial rRNA genes. The following primers and TaqMan probe were used for detection of Bifidobacteria rRNA: 5'-CGGGTGAGTAATGCGTGACC-3', 5'-TGATAGGACGCGACCCCA-3', and 5'-6FAM-CTCCTGGAAACGGGTG-QSY-3' (13). The following primers and TaqMan probe were used for detection of total eubacteria rRNA: 5'-TCCTACGGGAGGCAGCAGT-3', 5'-GGACTACCAGGGTATCTAATCCTGTT-3', and 5'-JUN-CGTATTACCGCGGCTGCTGGCAC-QSY-3' (24).

Bifidobacteria stimulation of murine T cells. Splenocytes and mesenteric lymph node cells were isolated from Jackson mice. Cells were suspended in medium (RPMI containing 10\% heat-inactivated FBS, $50 \mu \mathrm{M} \beta$-mercaptoethanol, $1 \mathrm{unit} / \mathrm{mL}$ penicillin, $1 \mu \mathrm{g} / \mathrm{mL}$ streptomycin, and $20 \mathrm{IU} / \mathrm{mL}$ recombinant murine IL-2 from R\&D Systems, Bio-Techne) at $2 \times 10^{6}$ viable cells $/ \mathrm{mL}$, and $4 \times 10^{6}$ cells were seeded per well in a 24-well plate. Bifidobacterium and Lactobacillus probiotic capsules were obtained from SeekingHealth. One capsule was resuspended in $10 \mathrm{~mL}$ of PBS and heated at $57^{\circ} \mathrm{C}$ for 1 hour. Splenocytes were stimulated with $160 \mu \mathrm{L}$ of the heat-killed resuspended bacteria (approximately 50-100 bacterial cfu per splenocyte) or left unstimulated. Cultures were maintained at $37^{\circ} \mathrm{C}$ at $5 \% \mathrm{CO}_{2}$ for 11 days. Fresh medium and IL-2 were added every other day. Cultures were then stained with anti-CD8a (APC-Cy7, 53-6.7, BioLegend), DAPI, and either PE-K ${ }^{\mathrm{b}}$-SIY dimer or a control PE-K $\mathrm{K}^{\mathrm{b}}$ dimer (in-house). Cells were analyzed on an LSRII flow cytometer (BD). Antigen-specific frequency was calculated by subtracting the noncognate background staining. DNA was extracted from $4 \times 10^{5}$ remaining cells using the DNeasy Blood and Tissue Kit (69504, QIAGEN). Extracted DNA was submitted to Adaptive Biotechnologies for survey-level TCR $\beta$ chain sequencing. 
Preparation of MHC-Ig dimers and nanoparticles. Soluble MHC-Ig dimers $\mathrm{K}^{\mathrm{b}}$-Ig (in-house) were prepared in-house and loaded with peptides (GenScript) as described (25). Anti-CD28 antibody was purchased from BioLegend (37.51). In-house particles were manufactured by directly conjugating peptide- $\mathrm{K}^{\mathrm{b}}$-Ig and antiCD28 to amine-coated, labeled, 80 - to $100-\mathrm{nm}$ super paramagnetic iron-oxide particles purchased from Micromod and functionalized according to the manufacturer's recommendations. Briefly, amines on particles reacted with Sulfo SMCC (Proteochem) and then were magnetically washed. Soluble peptide-MHC-Ig and anti-CD28 were modified with 2-iminothiolane $\mathrm{HCl}$ purchased from MilliporeSigma. Excess 2-iminothiolane was washed away from protein solution by a Vivaspin 20, $50 \mathrm{kDa}$ MWCO concentrator (GE Healthcare). Peptide-MHC-Ig and anti-CD28 were mixed to a 1:1 ratio and added to washed particles and mixed overnight at $25^{\circ} \mathrm{C}$. Particles were then washed and stored at $4{ }^{\circ} \mathrm{C}$. Nano-aAPCs were stored at a concentration of $5 \times 10^{12}$ particles $/ \mathrm{mL}$.

$T$ cell enrichment and expansion stimulations and cross-reactivity. Spleen and lymph nodes were harvested and isolated with CD8 kits (130-104-075, Miltenyi Biotec) as per the manufacturer's instructions. CD8 ${ }^{+} \mathrm{T}$ cells were incubated with nanoparticles at $10 \mu \mathrm{L}$ of particles per $10^{7} \mathrm{CD} 8^{+} \mathrm{T}$ cells at $4^{\circ} \mathrm{C}$ for 1 hour, and cells were washed over MS Miltenyi column. The positive fraction was eluted and plated at $2.5 \times 10^{5}$ cells/ $\mathrm{mL}$ and cultured for 7 days in 96-well round-bottom plates in complete RPMI 1640 medium supplemented with $10 \%$ human autologous serum and $3 \% \mathrm{~T}$ cell growth factor, a cytokine cocktail derived from stimulated PBMCs as described in the literature (12), in a humidified $5 \% \mathrm{CO}_{2}, 37^{\circ} \mathrm{C}$, incubator. Specificity of cytotoxic T lymphocytes was monitored on day 7, by FACS analysis following viability stain (L34970, Invitrogen, Thermo Fisher Scientific), dimeric MHC-Ig staining (PE, in-house), and anti-CD8a (APC, 53-6.7, BioLegend). The number of antigen-specific cells was calculated by multiplying the number of total live cells by the fractions of $\mathrm{CD} 8^{+}$and antigen-specific cells; the fraction of antigen-specific cells was calculated after subtracting the noncognate MHC staining from cognate MHC staining.

T cell cytokine response. After 7 days of stimulation, T cells were incubated with GolgiStop (554724, BD Biosciences) and GolgiPlug (555029, BD Biosciences) and anti-CD107a (FITC, 1D4B, BD Pharmingen) in RPMI 1640 medium supplemented with $10 \%$ FBS. RMA-S pulsed overnight at $25^{\circ} \mathrm{C}$ with peptide at 1 $\mathrm{mg} / \mathrm{mL}$ were washed and added to cells at a 1:2 ratio (RMA-S/T cells). Cells were then incubated at $37^{\circ} \mathrm{C}$ for 6 hours. Cells were washed and stained with anti-CD8a, (PerCP, 53-6.7, BioLegend) and viability stain (L34970, Thermo Fisher Scientific) for 15 minutes at $4^{\circ} \mathrm{C}$. Cells were then washed with PBS, and $100 \mu \mathrm{L}$ of Cytofix/Cytoperm Fixation and Permeabilization Solution (51-2090KZ, BD Biosciences) was added to the cells and incubated overnight at $4^{\circ} \mathrm{C}$. Cells were washed with Perm/Wash Buffer (51-2090KZ, BD Biosciences). Cells were stained with anti-IFN- $\gamma$ (PE, XMG1.2, BD Pharmingen), anti-IL-2 (APC, JES6$5 \mathrm{H} 4, \mathrm{BD}$ Pharmingen), and anti-TNF- $\alpha$ (PE-Cy7, MP6-XT22, BioLegend) for 1 hour at $4^{\circ} \mathrm{C}$. Cells were washed and read on an LSRII flow cytometer (BD). Background cytokine staining was accounted for by subtracting cytokine-positive cells in nonstimulated conditions from the restimulated cells.

$M D$ simulation setup. Each system consisted of a protein complex in one of the following 3 states: (a) the epitope only, (b) the epitope bound to $\mathrm{H} 2-\mathrm{K}^{\mathrm{b}} \mathrm{MHC}$ ( $\mathrm{K}^{\mathrm{b}}$ /epitope binary complex), and (c) $2 \mathrm{C} \mathrm{TCR}$ bound to the $\mathrm{H} 2-\mathrm{K}^{\mathrm{b}}$ /epitope complex ( $\mathrm{K}^{\mathrm{b}}$ /epitope/2C TCR ternary complex). The epitope sequence of each system was chosen to be either SIYRYYGL (SIY) or SVYRYYGL (SVY). The initial coordinates of H2-K $\mathrm{K}^{\mathrm{b}}$ and $2 \mathrm{C}$ TCR were taken from the x-ray crystal structure of each (Protein Data Bank [PDB] 3P9L, ref. 10; H2-K PDB 2OI9, ref. 11: 2C TCR). The initial configuration of the epitope was built based on the $\mathrm{OVA}_{257-264}$ epitope (SIINFEKL), which is complexed with $\mathrm{H} 2-\mathrm{K}^{\mathrm{b}}$ in the crystal structure. The OVA epitope was mutated to either SIY or SVY epitopes using VMD mutator plugin (26). The H2-K $/$ epitope/2C TCR complex was constructed in the following steps: the coordinates of $2 \mathrm{C} \mathrm{TCR}$ in the crystal structure were adjusted by aligning the $\alpha$-carbons of $\mathrm{H} 2-\mathrm{K}^{\mathrm{b}}$ and $\mathrm{MHC}$ protein between the 2 crystal structures; then the structures of $\mathrm{H} 2-\mathrm{K}^{\mathrm{b}}$, epitope, and $2 \mathrm{C}$ TCR were combined into one system. The protein complex was placed in the center of a rectangular box with a periodic boundary condition. The rest of the empty space of the box was filled with water molecules using the GROMACS tool solvate (27). The sizes of the simulation box for the protein complex in states 1,2 , and 3 were $60 \AA \times 60 \AA \times 60 \AA, 83 \AA \times 90 \AA \times 114 \AA$, and $86 \AA \times 90 \AA \times 145 \AA$, respectively. The length of each side of the box was set to be sufficiently large that proteins at the center of the box were separated at least $30 \AA$ away from their periodic images. The protonation states of His residues of the proteins were determined based on an optimal hydrogen bonding conformation, determined by the GROMACS tool $p d b 2 g m x$. His93 of the $\alpha$ chain of H2- $\mathrm{K}^{\mathrm{b}} \mathrm{MHC}$, and His 29 of the $\beta$ chain of $2 \mathrm{C}$ TCR, were singly protonated at the $\mathrm{N}^{\delta 1}$ atom. His 84 of the $\beta$ chain of $\mathrm{H} 2-\mathrm{K}^{\mathrm{b}}$ was doubly protonated at the $\mathrm{N}^{\delta 1}$ and 
the $\mathrm{N}^{\varepsilon 2}$ atoms. All other His residues were singly protonated at the $\mathrm{N}^{\varepsilon 2}$ atom. Standard protonation states were chosen for all other residues. $\mathrm{Na}^{+}$and $\mathrm{Cl}^{-}$ions were added in the solvent using the GROMACS tool genion, to neutralize the protein systems, as well as mimic the physiological ionic concentration, $150 \mathrm{mM}$. The CHARMM36 force field (28) was employed for the protein and ions and TIP3P model (29) for waters. The MD simulations were performed with GROMACS package version 5.14 (27). Long-ranged electrostatic interactions were treated with the Particle Mesh Ewald method (30). The cutoff distance for both the Lennard-Jones and the real space Coulomb interaction was set to be $12 \AA$. All covalent bonds involving the hydrogen atoms of the protein and water molecules were constrained by the LINC algorithm (31). Each system was minimized at $0 \mathrm{~K}$ for 1000 steps, then equilibrated in NPT (constant pressure and temperature) ensemble with a temperature of $300 \mathrm{~K}$ and a pressure of $1 \mathrm{~atm}$ for $1 \mathrm{~ns}$. The time step was set to be $2.0 \mathrm{fs}$. After the total volume of the system was stabilized in NPT ensemble, the system was equilibrated again in NVT (constant volume and temperature) ensemble at $300 \mathrm{~K}$ for $100 \mathrm{~ns}$. Then, the NVT simulation was extended for another $500 \mathrm{~ns}$ for the production run. The temperature and the pressure were controlled by Nose-Hoover (32) and Berendsen (33) schemes. The system coordinates were written every 100 ps during the production run; then all MD frames were clustered by the RMSD of the protein complex using gromos method (34). The RMSD cutoff value of each cluster was set to be $2.5 \AA$.

Free energy perturbation. The FEP calculation was performed to calculate the relative free energy changes of the systems in 3 states, while Val2 of the SIY epitope was mutated to Ile2. The hybrid structure and topology of each system were generated by PMX software (35). The alchemical transformation from Val2 to Ile2 was governed by the hybrid Hamiltonian $\mathbf{H}(\lambda)$, as a function of the coupling parameter $\lambda(10)$, where $\mathbf{H}(\lambda)=(1-\lambda) \times \mathbf{H}_{\mathrm{V}}+\lambda \times \mathbf{H}_{\mathrm{I}}+\mathbf{H}_{0}$.

$\mathbf{H}_{\mathrm{V}}$ and $\mathbf{H}_{1}$ are the Hamiltonians for the atoms of Val2 and Ile2, respectively, which undergo the alchemical transformation during the simulation, and $\mathbf{H}_{0}$ is the Hamiltonian for the rest of the system, with a fixed topology. Thirty-four $\lambda$-windows were generated between $\lambda=0$ and $\lambda=1$, where the $\lambda$-interval between neighboring windows was fixed to 0.04 between $\lambda=0.02$ and $\lambda=0.98$ and gradually decreased from $10^{-2}$ to $10^{-5}$, as $\lambda$ approaches both ends, $\lambda=0$ and $\lambda=1$. To circumvent numerical instability caused by insertion or deletion of atoms in the simulation, the soft-core potential (36) was applied to both the van der Waals and the electrostatic interactions for the atoms in the alchemical domain. The $\lambda$ value for the electrostatic interaction was fixed to 0 at the windows between $\lambda=0$ and $\lambda=0.1$, then rescaled linearly to $[0,1]$ at the windows between $\lambda=0.1$ and $\lambda=1$, while the $\lambda$ value for the van der Waals and the bonded interactions was set to be equal to that of the window. At each $\lambda$-window, the system was equilibrated for $100 \mathrm{ps}$, followed by the production run for $500 \mathrm{ps}$. The simulation was carried out sequentially from $\lambda=0$ to $\lambda=1$ so that the initial coordinates and velocities of each window were taken from the last MD frame of the previous window. The setup for the MD simulation part was the same used in the NPT simulation in the earlier section. All other FEP setups were the same used in our previous studies (37-40) on other MHC-epitope-TCR systems.

The free energy change $(\Delta G)$ was calculated using the thermodynamic integration method $(26,41,42)$, in the equation as follows (11):

$\Delta \mathbf{G}=\int_{0}^{1}\left\langle\left(\frac{\partial H(\lambda, \mathbf{x})}{\partial \lambda}\right)\right\rangle_{\lambda} d \lambda \quad$ (Equation 1),

where $\langle(\partial H(\lambda, \mathrm{x}) / \partial \lambda)\rangle_{\lambda}$ is the ensemble average of the derivative of the hybrid potential with respect to $\lambda$. $\partial H(\lambda, \mathrm{x}) / \partial \lambda$ was written every 0.1 ps. $\Delta G$ was calculated for each of 3 systems with different protein complexes as listed above $\left(\Delta G_{e p i}, \Delta G_{K b / e p i}\right.$, and $\left.\Delta G_{K b / e p i / T C R}\right)$. Statistical error of $\Delta G$ was estimated using the block average method by dividing the trajectory of each window into 4 consecutive blocks. To better sample the conformational change of the protein complex, $\Delta G_{K b / e p i}$ and $\Delta G_{K b / e p i / T C R}$ were averaged over 5 independent runs, starting from different initial configurations. Each initial configuration was taken from the frame at the center of each of the top 5 most populated clusters from the MD simulations, as shown in Supplemental Figure 3. $\Delta G$ of each individual run was weighted by the population of each cluster. Due to its small system size, $\Delta G_{e p i}$ was calculated from a single run. The calculation details are shown in Supplemental Table 2. The relative free energy changes for the 2 reaction steps during the formation of the protein complex, $\Delta \Delta G_{K b+e p i}$ and $\Delta \Delta G_{K b / e p i+T C R}$, defined in the main text, were calculated as follows $(26,27)$ :

$$
\begin{gathered}
\Delta \Delta G_{K b+e p i}=\Delta G_{K b / e p i}-\Delta G_{e p i} \\
\Delta \Delta G_{K b / e p i+T C R}=\Delta G_{k b / e p i / T C R}-\Delta G_{K b / e p i} \quad \text { (Equation 2) }
\end{gathered}
$$


Immunization assay. Recipient mice were injected intraperitoneally with $10 \mu \mathrm{g} / \mathrm{mouse}$ of anti-CD40 $\mathrm{mAb}$ (clone 3/23; BioLegend) and a day later immunized subcutaneously with $250 \mu \mathrm{g}$ as indicated of either ${ }^{\text {VVA- }} \mathrm{K}^{\mathrm{b}}$-Ig or ${ }^{\mathrm{SVY}-} \mathrm{K}^{\mathrm{b}}$-Ig dimer (14).

In vivo killing assay. Target cells were collected from splenocytes of naive mice, cleaned from erythrocytes by osmotic lysis, washed, and split into 2 populations. The control population was incubated at $37^{\circ} \mathrm{C}$ for 20 minutes and labeled with a high concentration of CFSE (Invitrogen, Thermo Fisher Scientific) (2.5 $\mu \mathrm{M})\left({ }^{\text {unlabeled- }}{ }^{-}\right.$FSE $^{\text {hi }}$ cells). The target population was pulsed with $1 \mu \mathrm{M}$ SVY, SIY, or OVA peptide and was labeled with a low concentration of CFSE $(0.25 \mu \mathrm{M})\left({ }^{\text {target- }}{ }^{-} F S E^{\text {lo }}\right.$ cells). The 2 populations were mixed together at a 1:1 ratio and intravenously injected into mice $\left(10 \times 10^{6} /\right.$ population/mouse $) 7$ days after immunization with $\mathrm{K}^{\mathrm{b}} \mathrm{SVY}$-Ig dimer, $\mathrm{K}^{\mathrm{b}} \mathrm{OVA}-\mathrm{Ig}$ dimer, or no immunization control mice. After 18 hours the mice were sacrificed and spleens harvested. The cell suspensions were analyzed by FACS for the presence of 2 differentially CFSE-labeled target populations. The recovery and percentage killing of the various CFSE-labeled, peptide-pulsed target cells were calculated as follows: $\%$ of in vivo killing $=100-([(\%$ specific peptide-pulsed cells in immunized/\% unspecific peptide-pulsed cells in immunized)/(\% specific peptide-pulsed cells in naive/\% unspecific peptide-pulsed cells in naive)] $\times 100$ ).

Cohousing experiment. Taconic and Jackson mice, 8 weeks old, were cohoused in fresh cages for 7 days. On day 7, age-matched Taconic mice, cohoused Taconic mice, and cohoused Jackson mice were sacrificed and spleens isolated for $\mathrm{CD}^{+} \mathrm{T}$ cell enrichment and expansion for $\mathrm{K}^{\mathrm{b} S V Y}$ and $\mathrm{K}^{\mathrm{b}} \mathrm{OVA} \mathrm{T}$ cell stimulations.

$T C R$ repertoire analysis. A custom $\mathrm{R}$ script was developed to analyze CDR3 data obtained from Adaptive Biotech based on both exact and homology overlaps between groups. Only sequences with a productive frequency more than $0.01 \%$ were included in the analysis. For exact overlap analysis, CDR3 reads were normalized within samples and then compared across all samples. First, the number of unique CDR3s and the overall contribution from shared sequences were compared. Next, PCA was performed and samples were plotted in terms of their top 2 principal components to examine sample clustering behavior in the lower dimensional space. Additionally, a heatmap representation was drawn using row-wise $Z$ scores. Finally, the Jensen Shannon Divergence was computed in terms of the Shannon's entropy of each CDR3 distribution as (43):

$D_{J S}(P \| Q)=H\left(\frac{1}{2} P+\frac{1}{2} Q\right)-\frac{1}{2}(H(P)+H(Q))$ (Equation 3),

where the Shannon's entropy in terms of the frequency of all $N$ unique CDR3s, $p(i)$ is (11):

$H(P)=-\sum_{i=1}^{N} p(i) \log _{2} p(i) \quad($ Equation 4$)$

For homology overlap analysis, the ImmunoMap algorithm was used (16). Briefly, pairwise distance scores between all unique CDR3s were calculated using a PAM10 substitution matrix, a gap opening penalty of 30 , and a gap extension penalty of 0 , and the ImmunoMap distance metric was calculated. Then, average hierarchical clustering was performed, and a homology threshold of 0.35 was used. Finally, dominant motifs were defined as homology clusters with a frequency greater than $3 \%$. Homology overlaps were represented in a similar manner as above.

Tumor growth and ACT. Jackson and Taconic mice, 8 weeks old, were injected with B16.SIY $\left(2 \times 10^{6}\right.$ cells $)$ or B16.F10 $\left(1 \times 10^{5}\right.$ cells $)$ in the flank and measured for tumor growth with calipers over time and monitored for survival. Jackson mice were injected subcutaneously with B16.SIY tumor cell line $\left(2 \times 10^{6}\right.$ cells). At 7 days after tumor injection, mice were partially ablated with 5 cGy to create room for adoptively transferred cells. On day 8, day 7-stimulated $\mathrm{K}^{\mathrm{b}} \mathrm{SIY}$ - or $\mathrm{K}^{\mathrm{b}} \mathrm{SVY}$-specific $\mathrm{CD} 8^{+} \mathrm{T}$ cells from Jackson mice were transferred into tumor-bearing animals by retro-orbital injection with $1.3 \times 10^{5}$ antigen-specific cells/ mouse. On day 8 and day 9, mice received IL-2 (30,000 units by intraperitoneal injection). Mice were measured 3 times/wk for tumor growth and survival, and tumor volume was reported in square millimeters.

TIL isolation. TILs were obtained from tumors by manual digestion and washing, followed by a density gradient centrifugation (Mouse, Lympholyte Cell Separation Media, CEDARLANE). Cells were counted and used for flow cytometry analysis. All cell lines underwent testing for mycoplasma contamination.

Data and materials availability. All data are available in the main text or the supplemental materials.

Statistics. Information on statistical tests is present in all figure legends. When making multiple comparisons, 1- and 2-way ANOVA were used. Bonferroni's posttests were performed when comparing all groups, and 
Dunnett's posttests were performed when the hypotheses being tested involved comparison against a single group. When comparing 2 groups, 1-tailed and 2-tailed $t$ tests were used, as indicated in figure legends. All data sets were assumed to fit a normal distribution. All graphs show mean and error bars represent SEM. All $n$ values are present within figure legends, and representative data include numbers of repeated experiments. Mice with outlier tumor size before the beginning of treatment were removed from the studies, with outlier size determined by no detected tumor or tumor larger than mean plus standard deviation from mean tumor size of all animals used in the experiment. Randomization was performed by cage in all animal studies. All statistical analysis was performed using GraphPad Prism software. $P$ value less than 0.05 was considered significant.

Study approval. The present studies in mice have been reviewed and approved by the Johns Hopkins University Institutional Review Board.

\section{Author contributions}

JJH and TAC conceived the project, and CAB, AI, JJH, TAC, JPS all designed and discussed the project. CAB, AI, JJH, JWH, WC, JGB, RS, FK, and TP all helped with the investigation. CAB, AI, and JJH acquired and analyzed data and helped write the manuscript. JWH, WC, RS, FK, and TP helped acquire data. JGB helped with analyzing data and writing the manuscript. AI conducted formal analysis and visualization of the TCR repertoire analysis. SL, DRB, and RZ conducted formal analysis for the TCR-modeling simulations. CAB led writing and AI, JJH, SL, RZ, TAC, and JPS edited the manuscript. RZ, TAC, and JPS supervised the project.

\section{Acknowledgments}

We would like to thank Jeffrey Weber and Binquan Luan for their various help with the modeling part of the work. We thank all members of the Chan lab for helpful discussions. We thank the Marie-Josée and Henry R. Kravis Center for Molecular Oncology for excellent suggestions. We also thank Memorial Sloan Kettering Cancer Center core laboratories, including Integrated Genomics Operations, Molecular Cytology, Cytogenetics, and Flow Cytometry cores. We thank the Sears lab for access to germ-free mice for experiments. We thank Eliot Sachsenmeier for his help. We thank the Johns Hopkins Bloomberg School of Public Health Bloomberg Flow Cytometry and Immunology Core and Dr. Hao Zhang. RZ acknowledges the support of the IBM Blue Gene Science Program (W1258591, W1464125, W1464164). This study was funded in part through the NIH/National Cancer Institute Cancer Center Support Grant P30 CA008748. We acknowledge funding sources, including Pershing Square Sohn Cancer Research grant (to TAC), the PaineWebber Chair (to TAC), Stand Up 2 Cancer (to TAC), NIH R01 CA205426 (to TAC), NIH R35 CA232097 (to TAC), the STARR Cancer Consortium (to TAC), and the Precision Immunotherapy Kidney Cancer Fund (to TAC, RJM). JWH thanks the NIH Cancer Nanotechnology Training Center at the Johns Hopkins Institute for NanoBioTechnology, the National Science Foundation Graduate Research Fellowship (DGE-1232825), and the ARCS Foundation for fellowship support. AI is supported by the National Science Foundation Graduate Research Fellowship. This work was funded by support from the NIH R01CA108835 to JPS, R21-EB023411 to JPS, R33-CA229042 to JPS, and P41-EB028239 to JPS; a grant from AstraZeneca (to JPS); and a gift from the Troper Wojcicki Foundation (to JPS).

Address correspondence to: Ruhong Zhou, 3000 Broadway, MC 3178, New York, New York 10027, USA. Phone: 914.945.3591; Email: rz24@columbia.edu. Or to: Timothy A. Chan, 1275 York Ave., New York, New York 10065, USA. Phone: 646.888.2765; Email: chant@mskcc.org. Or to: Jonathan P. Schneck, 733 N. Broadway, BRB 639, Baltimore, Maryland 21205, USA. Phone: 410.614.4589; Email: jschnec1@jhmi.edu.

RZ's present address is: Institute of Quantitative Biology, Zhejiang University, Hangzhou, China, and Department of Chemistry, Columbia University, New York, New York, USA.

1. Rizvi NA, et al. Cancer immunology. Mutational landscape determines sensitivity to PD-1 blockade in non-small cell lung cancer. Science. 2015;348(6230):124-128.

2. Gubin MM, et al. Checkpoint blockade cancer immunotherapy targets tumour-specific mutant antigens. Nature. 2014;515(7528):577-581.

3. Gros A, et al. Prospective identification of neoantigen-specific lymphocytes in the peripheral blood of melanoma patients. Nat Med. 2016;22(4):433-438.

4. Łuksza M, et al. A neoantigen fitness model predicts tumour response to checkpoint blockade immunotherapy. Nature. 
2017;551(7681):517-520.

5. Balachandran VP, et al. Identification of unique neoantigen qualities in long-term survivors of pancreatic cancer. Nature. 2017;551(7681):512-516.

6. Routy B, et al. Gut microbiome influences efficacy of PD-1-based immunotherapy against epithelial tumors. Science. 2018;359(6371):91-97.

7. Gopalakrishnan V, et al. Gut microbiome modulates response to anti-PD-1 immunotherapy in melanoma patients. Science. 2018;359(6371):97-103.

8. Iida N, et al. Commensal bacteria control cancer response to therapy by modulating the tumor microenvironment. Science. 2013;342(6161):967-970.

9. Viaud S, et al. The intestinal microbiota modulates the anticancer immune effects of cyclophosphamide. Science. 2013;342(6161):971-976.

10. Denton AE, et al. Affinity thresholds for naive CD8+ CTL activation by peptides and engineered influenza A viruses. JImmunol. 2011;187(11):5733-5744.

11. Colf LA, et al. How a single T cell receptor recognizes both self and foreign MHC. Cell. 2007;129(1):135-146.

12. Perica $\mathrm{K}$, et al. Enrichment and expansion with nanoscale artificial antigen presenting cells for adoptive immunotherapy. ACS Nano. 2015;9(7):6861-6871.

13. Sivan A, et al. Commensal Bifidobacterium promotes antitumor immunity and facilitates anti-PD-L1 efficacy. Science. 2015;350(6264):1084-1089.

14. Schütz C, Zoso A, Peng S, Bennett JD, Schneck JP, Oelke M. MHC-Ig induces memory T cell formation in vivo and inhibits tumour growth. Immun Inflamm Dis. 2014;2(3):181-192.

15. Blank C, et al. PD-L1/B7H-1 inhibits the effector phase of tumor rejection by $\mathrm{T}$ cell receptor (TCR) transgenic CD8+ T cells. Cancer Res. 2004;64(3):1140-1145.

16. Sidhom JW, Bessell CA, Havel JJ, Kosmides A, Chan TA, Schneck JP. ImmunoMap: a bioinformatics tool for T-cell repertoire analysis. Cancer Immunol Res. 2018;6(2):151-162.

17. Blank C, Brown I, Kacha AK, Markiewicz MA, Gajewski TF. ICAM-1 contributes to but is not essential for tumor antigen cross-priming and CD8+ T cell-mediated tumor rejection in vivo. J Immunol. 2005;174(6):3416-3420.

18. Matson V, et al. The commensal microbiome is associated with anti-PD-1 efficacy in metastatic melanoma patients. Science. 2018;359(6371):104-108.

19. Gil-Cruz C, et al. Microbiota-derived peptide mimics drive lethal inflammatory cardiomyopathy. Science. 2019;366(6467):881-886.

20. Ruff WE, et al. Pathogenic autoreactive T and B cells cross-react with mimotopes expressed by a common human gut commensal to trigger autoimmunity. Cell Host Microbe. 2019;26(1):100-113.e8.

21. Tanoue T, et al. A defined commensal consortium elicits CD8 T cells and anti-cancer immunity. Nature. 2019;565(7741):600-605

22. Routy B, Gopalakrishnan V, Daillère R, Zitvogel L, Wargo JA, Kroemer G. The gut microbiota influences anticancer immunosurveillance and general health. Nat Rev Clin Oncol. 2018;15(6):382-396.

23. Zitvogel L, Ma Y, Raoult D, Kroemer G, Gajewski TF. The microbiome in cancer immunotherapy: diagnostic tools and therapeutic strategies. Science. 2018;359(6382):1366-1370.

24. Haarman M, Knol J. Quantitative real-time PCR assays to identify and quantify fecal Bifidobacterium species in infants receiving a prebiotic infant formula. Appl Environ Microbiol. 2005;71(5):2318-2324.

25. Frenkel D, Smit B. Understanding Molecular Simulation: From Algorithms to Applications. Cambridge, Massachusetts, USA: Elsevier; 2001.

26. Humphrey W, Dalke A, Schulten K. VMD: visual molecular dynamics. J Mol Graph. 1996;14(1):33-38.

27. Abraham MJ, et al. GROMACS: High performance molecular simulations through multi-level parallelism from laptops to supercomputers. SoftwareX. 2015;1:19-25.

28. Best RB, et al. Optimization of the additive CHARMM all-atom protein force field targeting improved sampling of the backbone $\varphi, \psi$ and side-chain $\chi(1)$ and $\chi(2)$ dihedral angles. J Chem Theory Comput. 2012;8(9):3257-3273.

29. Jorgensen WL, Chandrasekhar J, Madura JD, Impey RW, Klein ML. Comparison of simple potential functions for simulating liquid water. J Chem Phys. 1983;79(2):926-935.

30. Darden T, York D, Pedersen L. Particle mesh Ewald: An N· $\log (\mathrm{N})$ method for Ewald sums in large systems. J Chem Phys. 1993;98(12):10089-10092.

31. Hess B, Bekker H, Berendsen HJC, Fraaije JGEM. LINCS: a linear constraint solver for molecular simulations. J Comput Chem. 1997;18(12):1463-1472.

32. Nosé S. A unified formulation of the constant temperature molecular dynamics methods. J Chem Phys. 1984;81(1):511-519.

33. Berendsen HJC, Postma JPM van, van Gunsteren WF, DiNola A, Haak JR. Molecular dynamics with coupling to an external bath. J Chem Phys. 1984;81(8):3684-3690.

34. González-Alemán R, Hernández-Castillo D, Caballero J, Montero-Cabrera LA. Quality threshold clustering of molecular dynamics: a word of caution. J Chem Inf Model. 2020;60(2):467-472.

35. Gapsys V, Michielssens S, Seeliger D, de Groot BL. pmx: automated protein structure and topology generation for alchemical perturbations. J Comput Chem. 2015;36(5):348-354.

36. Beutler TC, Mark AE, van Schaik RC, Gerber PR, van Gunsteren WF. Avoiding singularities and numerical instabilities in free energy calculations based on molecular simulations. Chem Phys Lett. 1994;222(6):529-539.

37. Xia Z, et al. The complex and specific pMHC interactions with diverse HIV-1 TCR clonotypes reveal a structural basis for alterations in CTL function. Sci Rep. 2014;4:4087.

38. Hölzemer A, et al. Selection of an HLA-C*03:04-restricted HIV-1 p24 gag sequence variant is associated with viral escape from KIR2DL3+ natural killer cells: data from an observational cohort in South Africa. PLoS Med. 2015;12(11):e1001900; discussion e1001900.

39. Chowell D, et al. Patient HLA class I genotype influences cancer response to checkpoint blockade immunotherapy. Science. 2018;359(6375):582-587.

40. Joglekar AV, et al. T cell receptors for the HIV KK10 epitope from patients with differential immunologic control are functionally indistinguishable. Proc Natl Acad Sci U S A. 2018;115(8):1877-1882. 
41. Kirkwood JG. Statistical mechanics of fluid mixtures. J Chem Phys. 1935;3(5):300-313.

42. Straatsma TP, McCammon JA. Multiconfiguration thermodynamic integration. J Chem Phys. 1991;95(2):1175-1188.

43. Russ BE, Denton AE, Hatton L, Croom H, Olson MR, Turner SJ. Defining the molecular blueprint that drives CD8(+) T cell differentiation in response to infection. Front Immunol. 2012;3:371. 